Supporting Information (33 pages)

\title{
Dizirconium Complexes Supported by Preorganized Binucleating Bis(amidinates)
}

John R. Hagadorn*, Michael J. McNevin, Grant Wiedenfeld, and Richard Shoemaker

Department of Chemistry and Biochemistry, University of Colorado, Boulder, Colorado 803090215

\section{Table of Contents}

\section{Crystal Structure of $\mathbf{1}$}

Figure S1. Drawing of molecular structure

Table S1. Atomic Coordinates and $\mathrm{U}_{\mathrm{eq}}$ Values

Table S2. Calculated coordinates of $\mathrm{H}$-atoms

Table S3. Anisotropic Thermal Parameters

Table S4. Bond Lengths

\section{$\underline{\text { Page }}$}

Table S5. Bond Angles

$\mathrm{S} 2$

S3

S4

S5

S6

S7-8

2. Crystal Structure of $\mathbf{3}$

Figure S2. Drawing of molecular structure

S9

Table S6. Atomic Coordinates and $\mathrm{U}_{\mathrm{eq}}$ Values

S10

Table S7. Calculated coordinates of $\mathrm{H}$-atoms

S11

Table S8. Anisotropic Thermal Parameters

$\mathrm{S} 12$

Table S9. Bond Lengths

S13-14

Table S10. Bond Angles

S15-18

3. Crystal Structure of 6

Figure S3. Drawing of molecular structure

Table S11. Atomic Coordinates and $\mathrm{U}_{\mathrm{eq}}$ Values

S20

Table S12. Calculated and refined coordinates of $\mathrm{H}$-atoms

S21-22

Table S13. Anisotropic Thermal Parameters

$\mathrm{S} 23$

Table S14. Bond Lengths

S24-26

Table S15. Bond Angles

S27-33 
Figure S1. Molecular structure of 1.drawn with 50\% thermal ellipsoids.

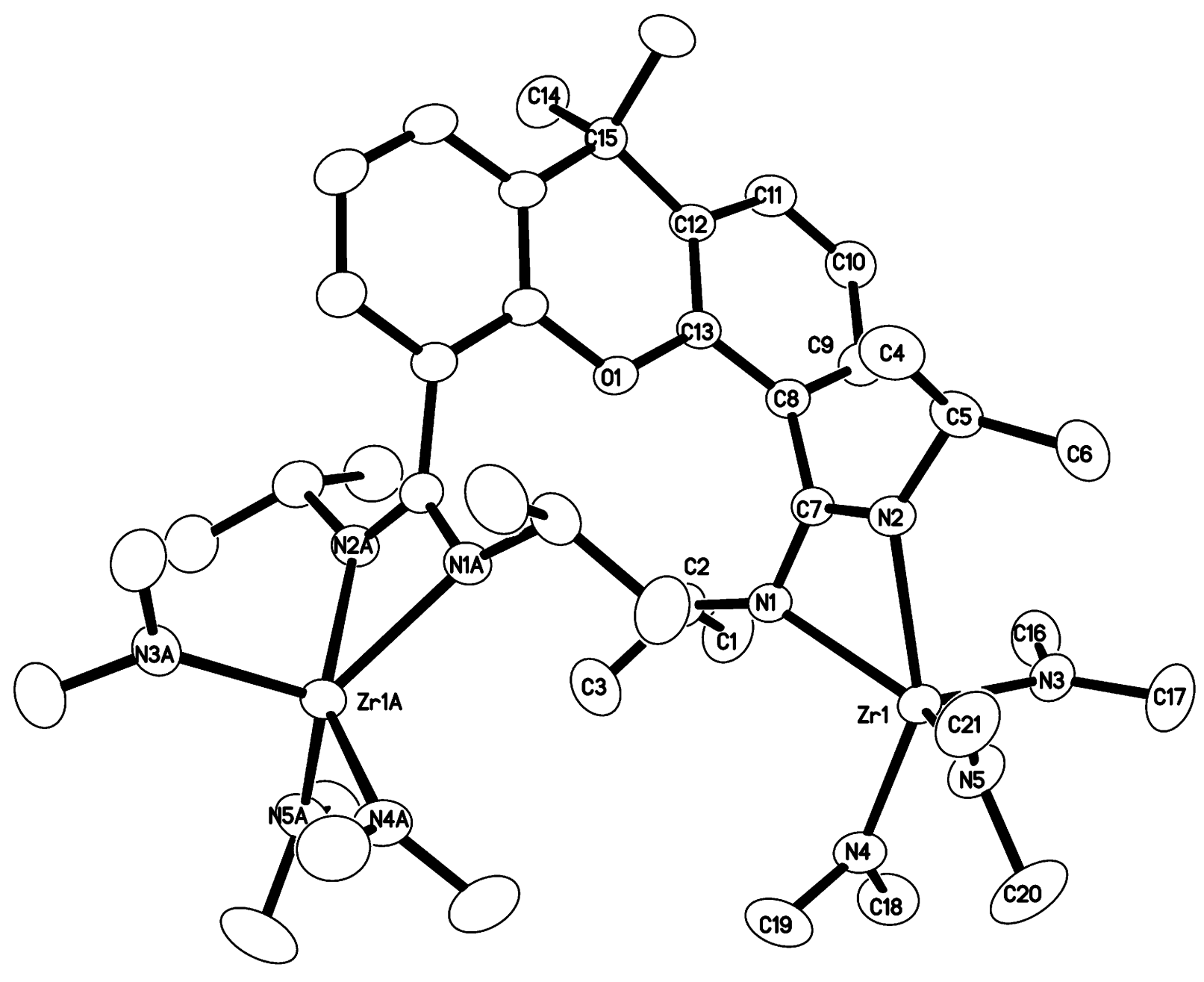


Table S1. Atomic Coordinates and $\mathrm{U}_{\mathrm{eq}}$ Values for $\mathbf{1}$

\begin{tabular}{|c|c|c|c|c|}
\hline & $\mathrm{x}$ & y & z & $\mathrm{U}(\mathrm{eq})$ \\
\hline $\operatorname{Zr}(1)$ & $9837(1)$ & $3000(1)$ & $1332(1)$ & $24(1)$ \\
\hline$O(1)$ & $5412(1)$ & 0 & 1667 & $26(1)$ \\
\hline$N(1)$ & $8081(1)$ & $1566(1)$ & $1100(1)$ & $26(1)$ \\
\hline $\mathrm{N}(2)$ & $8176(1)$ & $2698(1)$ & $1823(1)$ & $27(1)$ \\
\hline $\mathrm{N}(3)$ & $10277(2)$ & $4527(1)$ & $939(1)$ & $33(1)$ \\
\hline$N(4)$ & $10732(2)$ & $2423(2)$ & $837(1)$ & $35(1)$ \\
\hline $\mathrm{N}(5)$ & $10796(1)$ & $3298(2)$ & $2066(1)$ & $36(1)$ \\
\hline$C(1)$ & $7842(2)$ & $1287(2)$ & $62(1)$ & $44(1)$ \\
\hline$C(2)$ & $7492(2)$ & $726(2)$ & $642(1)$ & $31(1)$ \\
\hline C (3) & 7767 (2) & $-249(2)$ & $704(1)$ & $47(1)$ \\
\hline$C(4)$ & $7066(2)$ & $2553(2)$ & $2685(1)$ & $44(1)$ \\
\hline$C(5)$ & $7746(2)$ & $3303(2)$ & $2178(1)$ & $33(1)$ \\
\hline$C(6)$ & $8761(2)$ & $4449(2)$ & $2379(1)$ & $47(1)$ \\
\hline$C(7)$ & $7544(1)$ & $2001(2)$ & $1405(1)$ & $24(1)$ \\
\hline $\mathrm{C}(8)$ & $6382(1)$ & $1840(2)$ & $1245(1)$ & $25(1)$ \\
\hline$C(9)$ & $6360(2)$ & $2719(2)$ & $938(1)$ & $32(1)$ \\
\hline$C(10)$ & $5319(2)$ & $2691(2)$ & $836(1)$ & $36(1)$ \\
\hline C (11) & $4314(2)$ & $1810(2)$ & $1069(1)$ & $33(1)$ \\
\hline C (12) & $4292(2)$ & $902(2)$ & $1376(1)$ & $25(1)$ \\
\hline C (13) & $5339(1)$ & $909(2)$ & $1430(1)$ & $23(1)$ \\
\hline$C(14)$ & $2223(2)$ & $-619(2)$ & $1225(1)$ & $39(1)$ \\
\hline$C(16)$ & $9757(2)$ & $4582(2)$ & $405(1)$ & $42(1)$ \\
\hline C (17) & $11129(2)$ & $5673(2)$ & $1133(1)$ & $51(1)$ \\
\hline C (18) & $11258(2)$ & $2833(3)$ & $285(1)$ & $47(1)$ \\
\hline C (19) & $10912(3)$ & $1502(3)$ & $1036(1)$ & $54(1)$ \\
\hline C (20) & $12035(2)$ & $3743(3)$ & $2022(1)$ & $60(1)$ \\
\hline C (21) & $10446(2)$ & $3056(2)$ & $2652(1)$ & $43(1)$ \\
\hline$C(15)$ & $3191(2)$ & 0 & 1667 & $26(1)$ \\
\hline
\end{tabular}


Table S2. Calculated coordinates of $\mathrm{H}$-atoms for $\mathbf{1}$

\begin{tabular}{|c|c|c|c|c|}
\hline & $x$ & $\mathrm{y}$ & z & $\mathrm{U}(\mathrm{eq})$ \\
\hline $\mathrm{H}(1 \mathrm{~A})$ & 7615 & 1877 & 26 & 66 \\
\hline $\mathrm{H}(1 \mathrm{~B})$ & 7457 & 699 & -233 & 66 \\
\hline $\mathrm{H}(1 \mathrm{C})$ & 8682 & 1648 & 18 & 66 \\
\hline $\mathrm{H}(2)$ & 6639 & 394 & 687 & 40 \\
\hline $\mathrm{H}(3 \mathrm{~A})$ & 8598 & 61 & 652 & 70 \\
\hline $\mathrm{H}(3 \mathrm{~B})$ & 7340 & -842 & 416 & 70 \\
\hline $\mathrm{H}(3 \mathrm{C})$ & 7538 & -591 & 1083 & 70 \\
\hline $\mathrm{H}(4 \mathrm{~A})$ & 6420 & 1822 & 2547 & 66 \\
\hline $\mathrm{H}(4 \mathrm{~B})$ & 6765 & 2962 & 2911 & 66 \\
\hline $\mathrm{H}(4 \mathrm{C})$ & 7575 & 2396 & 2921 & 66 \\
\hline H (5) & 7220 & 3467 & 1944 & 43 \\
\hline $\mathrm{H}(6 \mathrm{~A})$ & 9261 & 4299 & 2627 & 70 \\
\hline $\mathrm{H}(6 \mathrm{~B})$ & 8469 & 4883 & 2589 & 70 \\
\hline $\mathrm{H}(6 \mathrm{C})$ & 9205 & 4901 & 2049 & 70 \\
\hline $\mathrm{H}(9)$ & 7060 & 3344 & 797 & 38 \\
\hline $\mathrm{H}(10)$ & 5301 & 3270 & 609 & 43 \\
\hline $\mathrm{H}(11)$ & 3614 & 1821 & 1018 & 39 \\
\hline $\mathrm{H}(14 \mathrm{~A})$ & 2435 & -1047 & 959 & 58 \\
\hline $\mathrm{H}(14 \mathrm{~B})$ & 2114 & -47 & 1015 & 58 \\
\hline $\mathrm{H}(14 \mathrm{C})$ & 1502 & -1158 & 1419 & 58 \\
\hline $\mathrm{H}(16 \mathrm{~A})$ & 9399 & 5062 & 453 & 63 \\
\hline $\mathrm{H}(16 \mathrm{~B})$ & 9166 & 3801 & 291 & 63 \\
\hline $\mathrm{H}(16 \mathrm{C})$ & 10355 & 4919 & 111 & 63 \\
\hline $\mathrm{H}(17 \mathrm{~A})$ & 11760 & 6028 & 856 & 77 \\
\hline $\mathrm{H}(17 \mathrm{~B})$ & 11437 & 5613 & 1502 & 77 \\
\hline $\mathrm{H}(17 \mathrm{C})$ & 10766 & 6150 & 1173 & 77 \\
\hline $\mathrm{H}(18 \mathrm{~A})$ & 12090 & 3118 & 311 & 71 \\
\hline $\mathrm{H}(18 \mathrm{~B})$ & 11132 & 3461 & 161 & 71 \\
\hline $\mathrm{H}(18 \mathrm{C})$ & 10909 & 2198 & 10 & 71 \\
\hline $\mathrm{H}(19 \mathrm{~A})$ & 10543 & 846 & 774 & 81 \\
\hline $\mathrm{H}(19 \mathrm{~B})$ & 10573 & 1258 & 1416 & 81 \\
\hline $\mathrm{H}(19 \mathrm{C})$ & 11743 & 1778 & 1055 & 81 \\
\hline $\mathrm{H}(20 \mathrm{~A})$ & 12450 & 4462 & 2240 & 89 \\
\hline $\mathrm{H}(20 \mathrm{~B})$ & 12270 & 3896 & 1622 & 89 \\
\hline $\mathrm{H}(20 \mathrm{C})$ & 12220 & 3172 & 2173 & 89 \\
\hline $\mathrm{H}(21 \mathrm{~A})$ & 10617 & 2471 & 2797 & 65 \\
\hline $\mathrm{H}(21 \mathrm{~B})$ & 9614 & 2768 & 2682 & 65 \\
\hline $\mathrm{H}(21 \mathrm{C})$ & 10868 & 3766 & 2876 & 65 \\
\hline
\end{tabular}


Table S3. Anisotropic thermal parameters for $\mathbf{1}$

\begin{tabular}{|c|c|c|c|c|c|c|}
\hline & U11 & $\mathrm{U} 22$ & U33 & U23 & U13 & U12 \\
\hline $\operatorname{Zr}(1)$ & $22(1)$ & $28(1)$ & $24(1)$ & $2(1)$ & $0(1)$ & $12(1)$ \\
\hline$O(1)$ & $22(1)$ & $28(1)$ & $29(1)$ & $6(1)$ & $3(1)$ & $14(1)$ \\
\hline $\mathrm{N}(1)$ & $23(1)$ & $30(1)$ & $26(1)$ & $-2(1)$ & $0(1)$ & $13(1)$ \\
\hline$N(2)$ & $24(1)$ & $32(1)$ & $24(1)$ & $-1(1)$ & $1(1)$ & $15(1)$ \\
\hline$N(3)$ & $29(1)$ & $31(1)$ & $35(1)$ & $4(1)$ & $0(1)$ & $12(1)$ \\
\hline $\mathrm{N}(4)$ & $29(1)$ & $42(1)$ & $37(1)$ & $3(1)$ & $2(1)$ & $21(1)$ \\
\hline $\mathrm{N}(5)$ & $27(1)$ & $42(1)$ & $32(1)$ & $6(1)$ & $-2(1)$ & $12(1)$ \\
\hline$C(1)$ & $46(1)$ & $45(1)$ & $27(1)$ & $-2(1)$ & $-4(1)$ & $13(1)$ \\
\hline$C(2)$ & $28(1)$ & $32(1)$ & $29(1)$ & $-5(1)$ & $1(1)$ & $11(1)$ \\
\hline$C(3)$ & $59(2)$ & $39(1)$ & $47(1)$ & $-7(1)$ & $3(1)$ & $28(1)$ \\
\hline$C(4)$ & $51(1)$ & $56(1)$ & $31(1)$ & $-2(1)$ & $10(1)$ & $30(1)$ \\
\hline$C(5)$ & $37(1)$ & $40(1)$ & $29(1)$ & $-5(1)$ & $1(1)$ & $24(1)$ \\
\hline$C(6)$ & $53(1)$ & $41(1)$ & $49(1)$ & $-14(1)$ & $-7(1)$ & $26(1)$ \\
\hline$C(7)$ & $24(1)$ & $26(1)$ & $24(1)$ & $4(1)$ & $3(1)$ & $14(1)$ \\
\hline$C(8)$ & $24(1)$ & $30(1)$ & $23(1)$ & $2(1)$ & $-1(1)$ & $15(1)$ \\
\hline$C(9)$ & $31(1)$ & $34(1)$ & $33(1)$ & $7(1)$ & $2(1)$ & $17(1)$ \\
\hline$C(10)$ & $39(1)$ & $40(1)$ & $36(1)$ & $8(1)$ & $-3(1)$ & $25(1)$ \\
\hline$C(11)$ & $31(1)$ & $41(1)$ & $34(1)$ & $4(1)$ & $-2(1)$ & $24(1)$ \\
\hline$C(12)$ & $25(1)$ & $33(1)$ & $23(1)$ & $-1(1)$ & $-2(1)$ & $18(1)$ \\
\hline$C(13)$ & $25(1)$ & $29(1)$ & $19(1)$ & $0(1)$ & $-1(1)$ & $16(1)$ \\
\hline$C(14)$ & $29(1)$ & $45(1)$ & $38(1)$ & $1(1)$ & $-10(1)$ & $15(1)$ \\
\hline$C(16)$ & $50(1)$ & $37(1)$ & $40(1)$ & $4(1)$ & $-6(1)$ & $22(1)$ \\
\hline$C(17)$ & $45(1)$ & $34(1)$ & $58(1)$ & $5(1)$ & $-13(1)$ & $8(1)$ \\
\hline$C(18)$ & $51(1)$ & $60(2)$ & $36(1)$ & $0(1)$ & $9(1)$ & $32(1)$ \\
\hline C (19) & $57(2)$ & $62(2)$ & $62(2)$ & $11(1)$ & $12(1)$ & $46(1)$ \\
\hline$C(20)$ & $27(1)$ & $81(2)$ & $51(1)$ & $4(1)$ & $-7(1)$ & $13(1)$ \\
\hline$C(21)$ & $38(1)$ & $52(1)$ & $29(1)$ & $4(1)$ & $-6(1)$ & $15(1)$ \\
\hline
\end{tabular}


Table S4. Bond lengths for 1

\begin{tabular}{ll}
\hline $\operatorname{Zr}(1)-\mathrm{N}(3)$ & $2.0409(17)$ \\
$\operatorname{Zr}(1)-\mathrm{N}(5)$ & $2.0652(17)$ \\
$\operatorname{Zr}(1)-\mathrm{N}(4)$ & $2.0759(16)$ \\
$\operatorname{Zr}(1)-\mathrm{N}(1)$ & $2.2315(15)$ \\
$\operatorname{Zr}(1)-\mathrm{N}(2)$ & $2.3503(14)$ \\
$\operatorname{Zr}(1)-C(7)$ & $2.6664(15)$ \\
$\operatorname{Zr}(1)-\operatorname{Zr}(1) \# 1$ & $7.1190(3)$ \\
O $(1)-C(13) \# 1$ & $1.3824(18)$ \\
O $(1)-C(13)$ & $1.3824(18)$ \\
$N(1)-C(7)$ & $1.337(2)$ \\
$N(1)-C(2)$ & $1.469(2)$ \\
$N(2)-C(7)$ & $1.326(2)$ \\
$N(2)-C(5)$ & $1.464(2)$ \\
$N(3)-C(17)$ & $1.451(3)$ \\
$N(3)-C(16)$ & $1.455(3)$ \\
$N(4)-C(19)$ & $1.444(3)$ \\
$N(4)-C(18)$ & $1.446(3)$ \\
$N(5)-C(21)$ & $1.441(3)$ \\
$N(5)-C(20)$ & $1.457(3)$ \\
$C(1)-C(2)$ & $1.513(3)$ \\
$C(2)-C(3)$ & $1.527(3)$ \\
$C(4)-C(5)$ & $1.530(3)$ \\
$C(5)-C(6)$ & $1.526(3)$ \\
$C(7)-C(8)$ & $1.504(2)$ \\
$C(8)-C(9)$ & $1.391(2)$ \\
$C(8)-C(13)$ & $1.396(2)$ \\
$C(9)-C(10)$ & $1.393(3)$ \\
$C(10)-C(11)$ & $1.382(3)$ \\
$C(11)-C(12)$ & $1.400(2)$ \\
$C(12)-C(13)$ & $1.400(2)$ \\
$C(12)-C(15)$ & $1.520(2)$ \\
$C(14)-C(15)$ & $1.539(2)$ \\
$C(15)-C(12) \# 1$ & $1.520(2)$ \\
$C(15)-C(14) \# 1$ & $1.539(2)$ \\
& \\
\hline
\end{tabular}


Table S5. Bond angles for 1

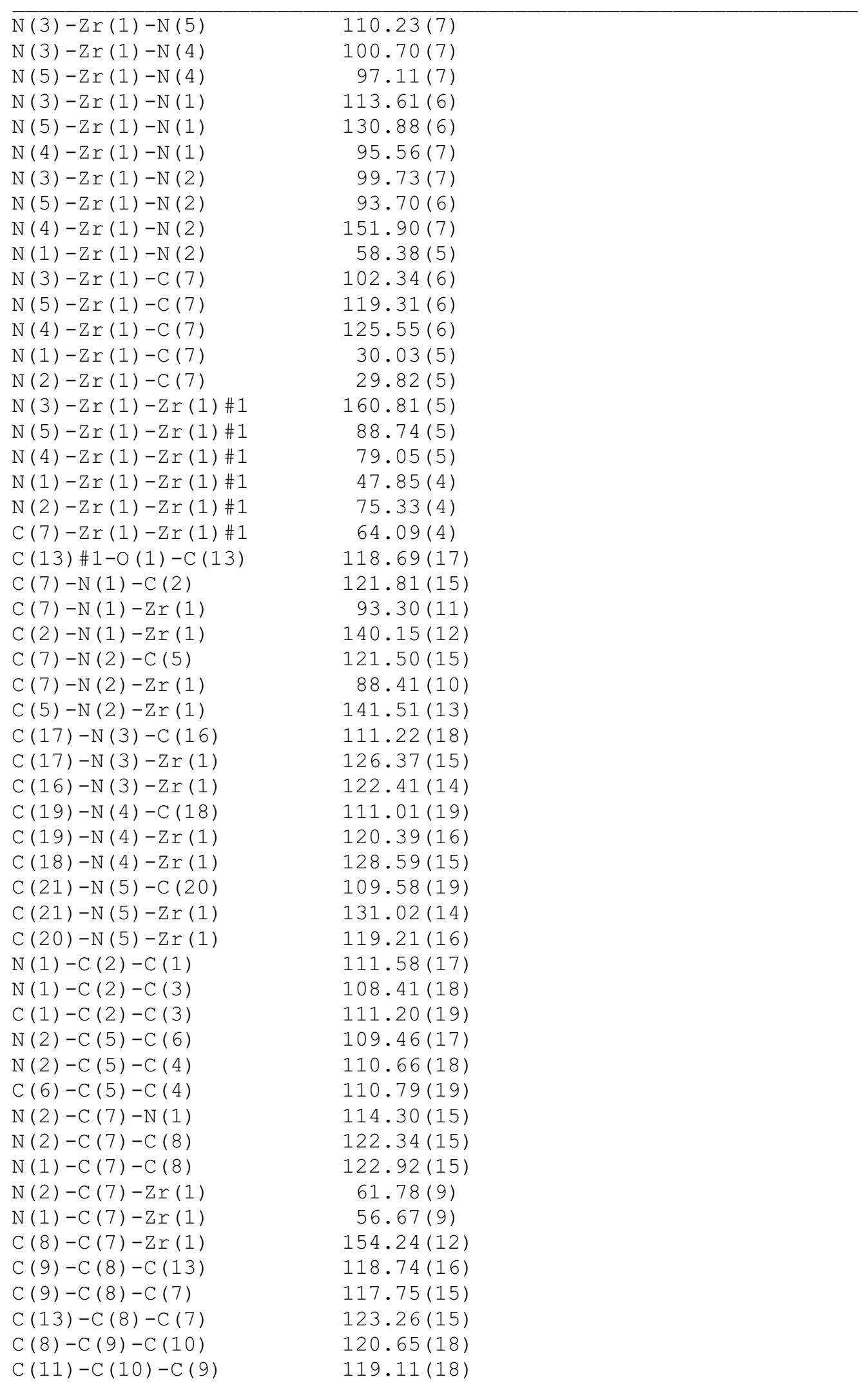




$\begin{array}{ll}C(10)-C(11)-C(12) & 122.23(18) \\ C(11)-C(12)-C(13) & 116.97(17) \\ C(11)-C(12)-C(15) & 120.82(16) \\ C(13)-C(12)-C(15) & 122.10(16) \\ O(1)-C(13)-C(8) & 115.53(14) \\ O(1)-C(13)-C(12) & 122.70(15) \\ C(8)-C(13)-C(12) & 121.77(16) \\ C(12) \# 1-C(15)-C(12) & 110.34(19) \\ C(12) \# 1-C(15)-C(14) \# 1 & 110.02(10) \\ C(12)-C(15)-C(14) \# 1 & 108.11(11) \\ C(12) \# 1-C(15)-C(14) & 108.11(11) \\ C(12)-C(15)-C(14) & 110.02(10) \\ C(14) \# 1-C(15)-C(14) & 110.2(2)\end{array}$


Figure S2. Molecular structure of $\mathbf{3}$ drawn with 50\% thermal ellipsoids.

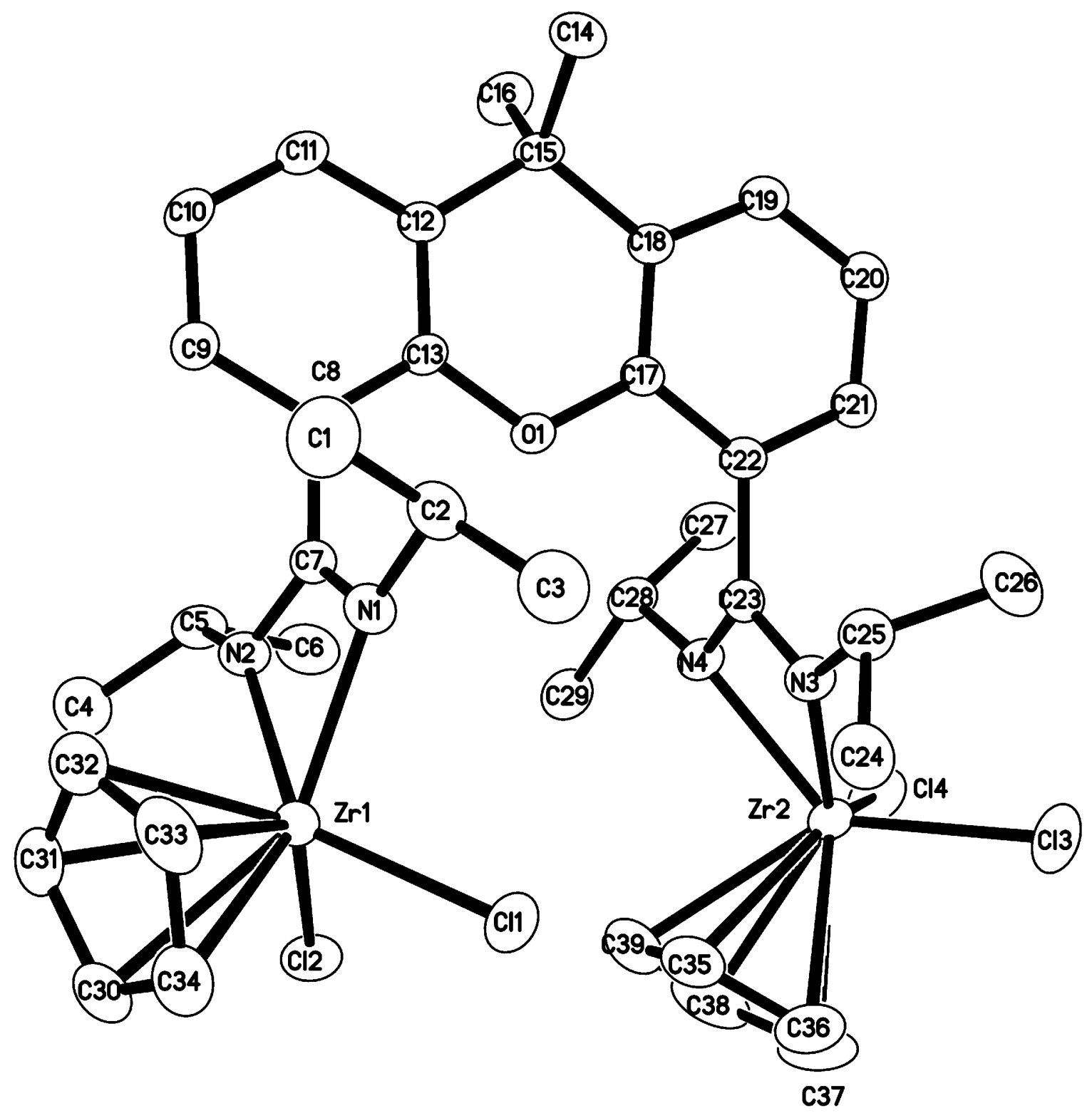


Table S6. Atomic Coordinates and $\mathrm{U}_{\mathrm{eq}}$ Values for $\mathbf{3}$

\begin{tabular}{|c|c|c|c|c|}
\hline & $x$ & $\mathrm{y}$ & $\mathrm{z}$ & $\mathrm{U}(\mathrm{eq})$ \\
\hline $\operatorname{Zr}(1)$ & $-8511(1)$ & $-4968(1)$ & $908(1)$ & $21(1)$ \\
\hline $\operatorname{Zr}(2)$ & $-5226(1)$ & $-6414(1)$ & $-1540(1)$ & $22(1)$ \\
\hline $\mathrm{Cl}(1)$ & $-6671(1)$ & $-5132(1)$ & $1166(1)$ & $33(1)$ \\
\hline $\mathrm{Cl}(2)$ & $-8716(1)$ & $-6272(1)$ & $592(1)$ & $31(1)$ \\
\hline $\mathrm{Cl}(3)$ & $-3332(1)$ & $-6285(1)$ & $-1587(1)$ & $44(1)$ \\
\hline $\mathrm{Cl}(4)$ & $-5220(1)$ & $-7157(1)$ & $-2672(1)$ & $37(1)$ \\
\hline$O(1)$ & $-7744(1)$ & $-4000(1)$ & $-1622(1)$ & $23(1)$ \\
\hline$N(1)$ & $-8167(2)$ & $-3959(1)$ & $228(1)$ & $24(1)$ \\
\hline $\mathrm{N}(2)$ & $-9476(2)$ & $-4713(1)$ & $-100(1)$ & $24(1)$ \\
\hline $\mathrm{N}(3)$ & $-5330(2)$ & $-5198(1)$ & $-1332(1)$ & $25(1)$ \\
\hline$N(4)$ & $-6542(2)$ & $-5761(1)$ & $-2013(1)$ & $24(1)$ \\
\hline$C(1)$ & $-8206(3)$ & $-2622(2)$ & $560(3)$ & $49(1)$ \\
\hline$C(2)$ & $-7587(2)$ & $-3253(1)$ & $196(2)$ & $30(1)$ \\
\hline$C(3)$ & $-6520(3)$ & $-3332(2)$ & $558(2)$ & $45(1)$ \\
\hline$C(4)$ & $-11084(2)$ & $-5446(2)$ & $-228(2)$ & $37(1)$ \\
\hline$C(5)$ & $-10223(2)$ & $-5035(1)$ & $-637(2)$ & $27(1)$ \\
\hline$C(6)$ & $-9638(3)$ & $-5528(2)$ & $-1191(2)$ & $35(1)$ \\
\hline$C(7)$ & $-8915(2)$ & $-4110(1)$ & $-271(1)$ & $22(1)$ \\
\hline$C(8)$ & $-9192(2)$ & $-3609(1)$ & $-904(2)$ & $23(1)$ \\
\hline$C(9)$ & $-10093(2)$ & $-3178(2)$ & $-818(2)$ & $31(1)$ \\
\hline$C(10)$ & $-10380(2)$ & $-2666(2)$ & $-1355(2)$ & $33(1)$ \\
\hline$C(11)$ & $-9762(2)$ & $-2574(2)$ & $-1980(2)$ & $29(1)$ \\
\hline C (12) & $-8853(2)$ & $-2998(1)$ & $-2098(1)$ & $23(1)$ \\
\hline$C(13)$ & $-8594(2)$ & $-3526(1)$ & $-1560(2)$ & $22(1)$ \\
\hline$C(14)$ & $-7780(3)$ & $-2038(1)$ & $-2751(2)$ & $35(1)$ \\
\hline$C(15)$ & $-8168(2)$ & $-2850(1)$ & $-2780(1)$ & $24(1)$ \\
\hline$C(16)$ & $-8816(2)$ & $-2967(2)$ & $-3506(2)$ & $34(1)$ \\
\hline$C(17)$ & $-7091(2)$ & $-3923(1)$ & $-2240(1)$ & $20(1)$ \\
\hline C (18) & $-7236(2)$ & $-3374(1)$ & $-2784(1)$ & $22(1)$ \\
\hline$C(19)$ & $-6503(2)$ & $-3318(1)$ & $-3361(2)$ & $26(1)$ \\
\hline$C(20)$ & $-5657(2)$ & $-3793(2)$ & $-3409(2)$ & $27(1)$ \\
\hline$C(21)$ & $-5549(2)$ & $-4353(1)$ & $-2881(2)$ & $25(1)$ \\
\hline$C(22)$ & $-6262(2)$ & $-4433(1)$ & $-2292(1)$ & $22(1)$ \\
\hline C (23) & $-6089(2)$ & $-5119(1)$ & $-1836(1)$ & $22(1)$ \\
\hline$C(24)$ & $-4490(3)$ & $-4734(2)$ & $-201(2)$ & $36(1)$ \\
\hline$C(25)$ & $-4759(2)$ & $-4558(2)$ & $-1011(2)$ & $28(1)$ \\
\hline$C(26)$ & $-3754(3)$ & $-4368(2)$ & $-1434(2)$ & $40(1)$ \\
\hline$C(27)$ & $-7024(3)$ & $-5801(2)$ & $-3382(2)$ & $35(1)$ \\
\hline$C(28)$ & $-7393(2)$ & $-5838(1)$ & $-2569(2)$ & $27(1)$ \\
\hline$C(29)$ & $-7966(2)$ & $-6566(2)$ & $-2419(2)$ & $35(1)$ \\
\hline$C(30)$ & $-9444(3)$ & $-5318(2)$ & $2088(2)$ & $35(1)$ \\
\hline$C(31)$ & $-10141(2)$ & $-4876(2)$ & $1672(2)$ & $35(1)$ \\
\hline$C(32)$ & $-9709(3)$ & $-4154(2)$ & $1637(2)$ & $39(1)$ \\
\hline$C(33)$ & $-8765(3)$ & $-4156(2)$ & $2032(2)$ & $40(1)$ \\
\hline$C(34)$ & $-8600(3)$ & $-4873(2)$ & $2310(2)$ & $40(1)$ \\
\hline$C(35)$ & $-5883(3)$ & $-6513(2)$ & $-224(2)$ & $39(1)$ \\
\hline$C(36)$ & $-4913(3)$ & $-6882(2)$ & $-250(2)$ & $42(1)$ \\
\hline$C(37)$ & $-5022(4)$ & $-7502(2)$ & $-717(2)$ & $50(1)$ \\
\hline$C(38)$ & $-6058(4)$ & $-7526(2)$ & $-964(2)$ & $52(1)$ \\
\hline$C(39)$ & $-6588(3)$ & $-6910(2)$ & $-663(2)$ & $47(1)$ \\
\hline
\end{tabular}


Table S7. Calculated coordinates of $\mathrm{H}$-atoms for $\mathbf{3}$

\begin{tabular}{|c|c|c|c|c|}
\hline & $x$ & y & z & $\mathrm{U}(\mathrm{eq})$ \\
\hline $\mathrm{H}(1 \mathrm{~A})$ & -8898 & -2588 & 326 & 74 \\
\hline $\mathrm{H}(1 \mathrm{~B})$ & -7830 & -2154 & 489 & 74 \\
\hline $\mathrm{H}(1 \mathrm{C})$ & -8286 & -2721 & 1096 & 74 \\
\hline $\mathrm{H}(2)$ & -7479 & -3124 & -343 & 36 \\
\hline $\mathrm{H}(3 \mathrm{~A})$ & -6605 & -3473 & 1085 & 68 \\
\hline $\mathrm{H}(3 \mathrm{~B})$ & -6145 & -2859 & 528 & 68 \\
\hline $\mathrm{H}(3 \mathrm{C})$ & -6120 & -3716 & 296 & 68 \\
\hline $\mathrm{H}(4 \mathrm{~A})$ & -10778 & -5847 & 70 & 56 \\
\hline $\mathrm{H}(4 \mathrm{~B})$ & -11576 & -5653 & -593 & 56 \\
\hline $\mathrm{H}(4 \mathrm{C})$ & -11456 & -5102 & 104 & 56 \\
\hline $\mathrm{H}(5)$ & -10551 & -4619 & -924 & 33 \\
\hline $\mathrm{H}(6 \mathrm{~A})$ & -9142 & -5227 & -1478 & 53 \\
\hline $\mathrm{H}(6 \mathrm{~B})$ & -10140 & -5761 & -1533 & 53 \\
\hline $\mathrm{H}(6 \mathrm{C})$ & -9257 & -5914 & -916 & 53 \\
\hline $\mathrm{H}(9)$ & -10514 & -3238 & -384 & 37 \\
\hline $\mathrm{H}(10)$ & -10998 & -2379 & -1294 & 39 \\
\hline $\mathrm{H}(11)$ & -9954 & -2213 & -2343 & 35 \\
\hline $\mathrm{H}(14 \mathrm{~A})$ & -7399 & -1954 & -2282 & 52 \\
\hline $\mathrm{H}(14 \mathrm{~B})$ & -8381 & -1702 & -2776 & 52 \\
\hline $\mathrm{H}(14 \mathrm{C})$ & -7314 & -1944 & -3176 & 52 \\
\hline $\mathrm{H}(16 \mathrm{~A})$ & -8386 & -2844 & -3943 & 52 \\
\hline $\mathrm{H}(16 \mathrm{~B})$ & -9435 & -2646 & -3495 & 52 \\
\hline $\mathrm{H}(16 \mathrm{C})$ & -9038 & -3486 & -3538 & 52 \\
\hline $\mathrm{H}(19)$ & -6587 & -2943 & -3729 & 31 \\
\hline $\mathrm{H}(20)$ & -5156 & -3738 & -3798 & 32 \\
\hline $\mathrm{H}(21)$ & -4979 & -4688 & -2920 & 30 \\
\hline $\mathrm{H}(24 \mathrm{~A})$ & -5129 & -4869 & 70 & 55 \\
\hline $\mathrm{H}(24 \mathrm{~B})$ & -4174 & -4297 & 34 & 55 \\
\hline $\mathrm{H}(24 \mathrm{C})$ & -3994 & -5148 & -185 & 55 \\
\hline H (25) & -5230 & -4116 & -1021 & 33 \\
\hline $\mathrm{H}(26 \mathrm{~A})$ & -3255 & -4778 & -1385 & 60 \\
\hline $\mathrm{H}(26 \mathrm{~B})$ & -3447 & -3916 & -1223 & 60 \\
\hline $\mathrm{H}(26 \mathrm{C})$ & -3915 & -4289 & -1964 & 60 \\
\hline $\mathrm{H}(27 \mathrm{~A})$ & -6742 & -5306 & -3487 & 53 \\
\hline $\mathrm{H}(27 \mathrm{~B})$ & -7616 & -5900 & -3717 & 53 \\
\hline $\mathrm{H}(27 \mathrm{C})$ & -6478 & -6173 & -3465 & 53 \\
\hline $\mathrm{H}(28)$ & -7900 & -5423 & -2487 & 33 \\
\hline $\mathrm{H}(29 \mathrm{~A})$ & -7482 & -6982 & -2489 & 52 \\
\hline $\mathrm{H}(29 \mathrm{~B})$ & -8554 & -6615 & -2768 & 52 \\
\hline $\mathrm{H}(29 \mathrm{C})$ & -8230 & -6568 & -1903 & 52 \\
\hline $\mathrm{H}(30)$ & -9531 & -5829 & 2199 & 49 \\
\hline H (31) & -10782 & -5034 & 1454 & 49 \\
\hline $\mathrm{H}(32)$ & -10010 & -3740 & 1389 & 55 \\
\hline $\mathrm{H}(33)$ & -8313 & -3743 & 2100 & 55 \\
\hline $\mathrm{H}(34)$ & -8017 & -5031 & 2599 & 55 \\
\hline $\mathrm{H}(35)$ & -6030 & -6071 & 46 & 55 \\
\hline $\mathrm{H}(36)$ & -4294 & -6736 & 5 & 59 \\
\hline $\mathrm{H}(37)$ & -4485 & -7845 & -842 & 70 \\
\hline $\mathrm{H}(38)$ & -6354 & -7893 & -1281 & 73 \\
\hline $\mathrm{H}(39)$ & -7301 & -6786 & -746 & 66 \\
\hline
\end{tabular}


Table S8. Anisotropic thermal parameters for 3

\begin{tabular}{|c|c|c|c|c|c|c|}
\hline & $\mathrm{U} 11$ & U22 & U33 & U23 & $\mathrm{U} 13$ & U12 \\
\hline Zr(1) & $22(1)$ & $24(1)$ & $18(1)$ & $1(1)$ & $0(1)$ & $2(1)$ \\
\hline $\operatorname{Zr}(2)$ & $24(1)$ & $20(1)$ & $23(1)$ & $0(1)$ & $-2(1)$ & $4(1)$ \\
\hline $\mathrm{Cl}(1)$ & $24(1)$ & $42(1)$ & $31(1)$ & $1(1)$ & $-4(1)$ & $5(1)$ \\
\hline $\mathrm{Cl}(2)$ & $39(1)$ & $24(1)$ & $29(1)$ & $2(1)$ & $-5(1)$ & $2(1)$ \\
\hline $\mathrm{Cl}(3)$ & $25(1)$ & $44(1)$ & $64(1)$ & $-6(1)$ & $-2(1)$ & $4(1)$ \\
\hline Cl (4) & $43(1)$ & $36(1)$ & $32(1)$ & $-11(1)$ & $-5(1)$ & $14(1)$ \\
\hline O (1) & $25(1)$ & $22(1)$ & $21(1)$ & $4(1)$ & $5(1)$ & $7(1)$ \\
\hline$N(1)$ & $27(1)$ & $24(1)$ & $23(1)$ & $0(1)$ & $0(1)$ & $-3(1)$ \\
\hline $\mathrm{N}(2)$ & $27(1)$ & $23(1)$ & $22(1)$ & $2(1)$ & $-3(1)$ & $-1(1)$ \\
\hline N (3) & $28(1)$ & $22(1)$ & $24(1)$ & $-1(1)$ & $-2(1)$ & $1(1)$ \\
\hline N (4) & $24(1)$ & $20(1)$ & $26(1)$ & $0(1)$ & $-4(1)$ & $2(1)$ \\
\hline$C(1)$ & $51(2)$ & $30(2)$ & $67(2)$ & $-15(2)$ & $9(2)$ & $-2(1)$ \\
\hline$C(2)$ & $32(1)$ & $27(1)$ & $31(1)$ & $-2(1)$ & $1(1)$ & $-6(1)$ \\
\hline$C(3)$ & $36(2)$ & $42(2)$ & $57(2)$ & $2(2)$ & $-9(2)$ & $-13(1)$ \\
\hline$C(4)$ & $31(1)$ & $42(2)$ & $39(2)$ & $2(1)$ & $-5(1)$ & $-9(1)$ \\
\hline$C(5)$ & $29(1)$ & $25(1)$ & $27(1)$ & $4(1)$ & $-8(1)$ & $-2(1)$ \\
\hline$C(6)$ & $49(2)$ & $32(1)$ & 25 (1) & $-1(1)$ & $-5(1)$ & $-4(1)$ \\
\hline$C(7)$ & $26(1)$ & $21(1)$ & $19(1)$ & $1(1)$ & $3(1)$ & $4(1)$ \\
\hline$C(8)$ & $25(1)$ & $21(1)$ & $23(1)$ & $3(1)$ & $3(1)$ & $3(1)$ \\
\hline C (9) & $31(1)$ & $31(1)$ & $31(1)$ & $7(1)$ & $8(1)$ & $9(1)$ \\
\hline$C(10)$ & $30(1)$ & $32(1)$ & $37(2)$ & $8(1)$ & $5(1)$ & $12(1)$ \\
\hline C (11) & $31(1)$ & $26(1)$ & $32(1)$ & $7(1)$ & $1(1)$ & $8(1)$ \\
\hline$C(12)$ & $26(1)$ & $21(1)$ & $23(1)$ & $4(1)$ & $2(1)$ & $3(1)$ \\
\hline C (13) & $22(1)$ & $19(1)$ & $24(1)$ & $1(1)$ & $1(1)$ & $3(1)$ \\
\hline C (14) & $43(2)$ & $21(1)$ & $40(2)$ & $6(1)$ & $14(1)$ & $4(1)$ \\
\hline C (15) & $30(1)$ & $19(1)$ & $22(1)$ & $4(1)$ & $1(1)$ & $5(1)$ \\
\hline$C(16)$ & $36(1)$ & $42(2)$ & $25(1)$ & $3(1)$ & $-4(1)$ & $8(1)$ \\
\hline C (17) & $23(1)$ & $19(1)$ & $19(1)$ & $1(1)$ & $2(1)$ & $2(1)$ \\
\hline$C(18)$ & $25(1)$ & $19(1)$ & $22(1)$ & $1(1)$ & $1(1)$ & $2(1)$ \\
\hline C (19) & $30(1)$ & $24(1)$ & $23(1)$ & $2(1)$ & $2(1)$ & $2(1)$ \\
\hline C (20) & $29(1)$ & $29(1)$ & $24(1)$ & $0(1)$ & $5(1)$ & $2(1)$ \\
\hline C (21) & $26(1)$ & $26(1)$ & $23(1)$ & $2(1)$ & $5(1)$ & $4(1)$ \\
\hline C (22) & $25(1)$ & $21(1)$ & $21(1)$ & $1(1)$ & $1(1)$ & $1(1)$ \\
\hline C (23) & $22(1)$ & $22(1)$ & $22(1)$ & $0(1)$ & $5(1)$ & $3(1)$ \\
\hline C (24) & $37(1)$ & $42(2)$ & $29(1)$ & $-5(1)$ & $-3(1)$ & $-7(1)$ \\
\hline C (25) & $30(1)$ & $25(1)$ & $29(1)$ & $-2(1)$ & $-5(1)$ & $-3(1)$ \\
\hline$C(26)$ & $40(2)$ & $41(2)$ & $38(2)$ & $2(1)$ & $1(1)$ & $-15(1)$ \\
\hline C (27) & $43(2)$ & $33(1)$ & $29(1)$ & $-1(1)$ & $-9(1)$ & $2(1)$ \\
\hline C (28) & $27(1)$ & $23(1)$ & $32(1)$ & $0(1)$ & $-7(1)$ & $0(1)$ \\
\hline C (29) & $29(1)$ & $30(1)$ & $45(2)$ & $0(1)$ & $-8(1)$ & $-2(1)$ \\
\hline C (30) & $41(2)$ & $40(2)$ & $24(1)$ & $3(1)$ & $10(1)$ & $-1(1)$ \\
\hline C (31) & $26(1)$ & $51(2)$ & $27(1)$ & $-4(1)$ & $5(1)$ & $2(1)$ \\
\hline C (32) & $51(2)$ & $37(2)$ & $30(2)$ & $-1(1)$ & $18(1)$ & $14(1)$ \\
\hline C (33) & $51(2)$ & $41(2)$ & $27(1)$ & $-12(1)$ & $13(1)$ & $-9(1)$ \\
\hline C (34) & $36(2)$ & $61(2)$ & $22(1)$ & $-3(1)$ & $1(1)$ & $2(1)$ \\
\hline$C(35)$ & $52(2)$ & $35(1)$ & $30(2)$ & $5(1)$ & $8(1)$ & $4(1)$ \\
\hline$C(36)$ & $54(2)$ & $46(2)$ & $26(1)$ & $6(1)$ & $-7(1)$ & $10(2)$ \\
\hline C (37) & $83(3)$ & $29(1)$ & $37(2)$ & $9(1)$ & $5(2)$ & $19(2)$ \\
\hline C (38) & $82(3)$ & $34(2)$ & $40(2)$ & $7(1)$ & $5(2)$ & $-22(2)$ \\
\hline C (39) & $42(2)$ & $59(2)$ & $40(2)$ & $18(2)$ & $5(1)$ & $-8(2)$ \\
\hline
\end{tabular}


Table S9. Bond lengths for $\mathbf{3}$

\begin{tabular}{|c|c|}
\hline $\operatorname{Zr}(1)-\mathrm{N}(2)$ & $2.228(2)$ \\
\hline $\operatorname{Zr}(1)-\mathrm{N}(1)$ & $2.231(2)$ \\
\hline $\operatorname{Zr}(1)-\mathrm{Cl}(1)$ & $2.4138(7)$ \\
\hline $\operatorname{Zr}(1)-C l(2)$ & $2.4317(7)$ \\
\hline $\operatorname{Zr}(1)-C(32)$ & $2.490(3)$ \\
\hline $\operatorname{Zr}(1)-C(31)$ & $2.496(3)$ \\
\hline $\operatorname{Zr}(1)-C(30)$ & $2.502(3)$ \\
\hline $\operatorname{Zr}(1)-C(33)$ & $2.506(3)$ \\
\hline $\operatorname{Zr}(1)-C(34)$ & $2.512(3)$ \\
\hline $\operatorname{Zr}(1)-C(7)$ & $2.663(2)$ \\
\hline $\operatorname{Zr}(2)-\mathrm{N}(4)$ & $2.220(2)$ \\
\hline $\operatorname{Zr}(2)-\mathrm{N}(3)$ & $2.229(2)$ \\
\hline $\operatorname{Zr}(2)-\operatorname{Cl}(4)$ & $2.4256(7)$ \\
\hline $\operatorname{Zr}(2)-C l(3)$ & $2.4340(7)$ \\
\hline $\operatorname{Zr}(2)-C(37)$ & $2.465(3)$ \\
\hline $\operatorname{Zr}(2)-C(36)$ & $2.485(3)$ \\
\hline $\operatorname{Zr}(2)-C(38)$ & $2.490(3)$ \\
\hline $\operatorname{Zr}(2)-C(35)$ & $2.501(3)$ \\
\hline $\operatorname{Zr}(2)-C(39)$ & $2.504(3)$ \\
\hline $\operatorname{Zr}(2)-C(23)$ & $2.637(2)$ \\
\hline$O(1)-C(13)$ & $1.387(3)$ \\
\hline$O(1)-C(17)$ & $1.390(3)$ \\
\hline $\mathrm{N}(1)-\mathrm{C}(7)$ & 1.335 (3) \\
\hline $\mathrm{N}(1)-\mathrm{C}(2)$ & $1.475(3)$ \\
\hline $\mathrm{N}(2)-\mathrm{C}(7)$ & $1.338(3)$ \\
\hline$N(2)-C(5)$ & $1.472(3)$ \\
\hline $\mathrm{N}(3)-\mathrm{C}(23)$ & $1.331(3)$ \\
\hline $\mathrm{N}(3)-\mathrm{C}(25)$ & $1.481(3)$ \\
\hline$N(4)-C(23)$ & $1.334(3)$ \\
\hline$N(4)-C(28)$ & $1.479(3)$ \\
\hline$C(1)-C(2)$ & $1.529(4)$ \\
\hline$C(2)-C(3)$ & $1.516(4)$ \\
\hline$C(4)-C(5)$ & $1.514(4)$ \\
\hline$C(5)-C(6)$ & $1.527(4)$ \\
\hline$C(7)-C(8)$ & $1.489(3)$ \\
\hline$C(8)-C(9)$ & $1.398(4)$ \\
\hline$C(8)-C(13)$ & $1.407(4)$ \\
\hline$C(9)-C(10)$ & $1.381(4)$ \\
\hline$C(10)-C(11)$ & $1.377(4)$ \\
\hline$C(11)-C(12)$ & $1.407(3)$ \\
\hline$C(12)-C(13)$ & $1.391(3)$ \\
\hline$C(12)-C(15)$ & $1.523(4)$ \\
\hline$C(14)-C(15)$ & $1.547(4)$ \\
\hline$C(15)-C(18)$ & $1.520(3)$ \\
\hline$C(15)-C(16)$ & $1.554(4)$ \\
\hline $\mathrm{C}(17)-\mathrm{C}(18)$ & $1.400(3)$ \\
\hline$C(17)-C(22)$ & $1.405(3)$ \\
\hline$C(18)-C(19)$ & $1.395(4)$ \\
\hline$C(19)-C(20)$ & $1.383(4)$ \\
\hline$C(20)-C(21)$ & $1.388(4)$ \\
\hline$C(21)-C(22)$ & $1.399(3)$ \\
\hline
\end{tabular}


S14

$\begin{array}{ll}C(22)-C(23) & 1.498(3) \\ C(24)-C(25) & 1.520(4) \\ C(25)-C(26) & 1.528(4) \\ C(27)-C(28) & 1.528(4) \\ C(28)-C(29) & 1.527(4) \\ C(30)-C(34) & 1.402(5) \\ C(30)-C(31) & 1.407(5) \\ C(31)-C(32) & 1.416(5) \\ C(32)-C(33) & 1.397(5) \\ C(33)-C(34) & 1.402(5) \\ C(35)-C(39) & 1.393(5) \\ C(35)-C(36) & 1.407(5) \\ C(36)-C(37) & 1.402(5) \\ C(37)-C(38) & 1.397(6) \\ C(38)-C(39) & 1.408(6)\end{array}$


Table S10. Bond angles for 3

\begin{tabular}{|c|c|}
\hline$N(2)-\operatorname{Zr}(1)-N(1)$ & $60.10(8)$ \\
\hline $\mathrm{N}(2)-\operatorname{Zr}(1)-\mathrm{Cl}(1)$ & $135.94(6)$ \\
\hline $\mathrm{N}(1)-\operatorname{Zr}(1)-\mathrm{Cl}(1)$ & $90.69(6)$ \\
\hline$N(2)-\operatorname{Zr}(1)-C 1(2)$ & $87.32(6)$ \\
\hline $\mathrm{N}(1)-\operatorname{Zr}(1)-\mathrm{Cl}(2)$ & $133.14(6)$ \\
\hline $\mathrm{Cl}(1)-\operatorname{Zr}(1)-\mathrm{Cl}(2)$ & $91.79(3)$ \\
\hline$N(2)-\operatorname{Zr}(1)-C(32)$ & $87.69(10)$ \\
\hline$N(1)-\operatorname{Zr}(1)-C(32)$ & $85.68(10)$ \\
\hline $\mathrm{Cl}(1)-\operatorname{Zr}(1)-\mathrm{C}(32)$ & $124.83(9)$ \\
\hline $\operatorname{Cl}(2)-\operatorname{Zr}(1)-C(32)$ & $128.69(9)$ \\
\hline$N(2)-\operatorname{Zr}(1)-C(31)$ & $88.01(10)$ \\
\hline$N(1)-\operatorname{Zr}(1)-C(31)$ & $114.05(10)$ \\
\hline $\mathrm{Cl}(1)-\operatorname{Zr}(1)-\mathrm{C}(31)$ & $135.77(8)$ \\
\hline$C l(2)-\operatorname{Zr}(1)-C(31)$ & $95.79(8)$ \\
\hline$C(32)-\operatorname{Zr}(1)-C(31)$ & $33.00(11)$ \\
\hline$N(2)-\operatorname{Zr}(1)-C(30)$ & $117.89(10)$ \\
\hline$N(1)-\operatorname{Zr}(1)-C(30)$ & $139.25(9)$ \\
\hline $\mathrm{Cl}(1)-\operatorname{Zr}(1)-\mathrm{C}(30)$ & $105.81(8)$ \\
\hline $\operatorname{Cl}(2)-\operatorname{Zr}(1)-C(30)$ & $84.26(8)$ \\
\hline$C(32)-\operatorname{Zr}(1)-C(30)$ & $54.18(11)$ \\
\hline$C(31)-\operatorname{Zr}(1)-C(30)$ & $32.70(11)$ \\
\hline$N(2)-\operatorname{Zr}(1)-C(33)$ & $116.96(11)$ \\
\hline$N(1)-\operatorname{Zr}(1)-C(33)$ & $89.09(10)$ \\
\hline $\mathrm{Cl}(1)-\operatorname{Zr}(1)-\mathrm{C}(33)$ & $92.56(9)$ \\
\hline $\operatorname{Cl}(2)-\operatorname{Zr}(1)-C(33)$ & $137.47(8)$ \\
\hline$C(32)-\operatorname{Zr}(1)-C(33)$ & $32.48(12)$ \\
\hline$C(31)-\operatorname{Zr}(1)-C(33)$ & $54.21(11)$ \\
\hline$C(30)-\operatorname{Zr}(1)-C(33)$ & $53.93(11)$ \\
\hline $\mathrm{N}(2)-\operatorname{Zr}(1)-\mathrm{C}(34)$ & $139.89(10)$ \\
\hline$N(1)-\operatorname{Zr}(1)-C(34)$ & $119.69(11)$ \\
\hline $\mathrm{Cl}(1)-\operatorname{Zr}(1)-\mathrm{C}(34)$ & $82.07(8)$ \\
\hline $\mathrm{Cl}(2)-\operatorname{Zr}(1)-\mathrm{C}(34)$ & $106.98(9)$ \\
\hline$C(32)-\operatorname{Zr}(1)-C(34)$ & $53.87(12)$ \\
\hline$C(31)-\operatorname{Zr}(1)-C(34)$ & $54.04(11)$ \\
\hline$C(30)-\operatorname{Zr}(1)-C(34)$ & $32.46(11)$ \\
\hline$C(33)-\operatorname{Zr}(1)-C(34)$ & $32.45(12)$ \\
\hline$N(2)-\operatorname{Zr}(1)-C(7)$ & $30.11(8)$ \\
\hline$N(1)-\operatorname{Zr}(1)-C(7)$ & $30.02(8)$ \\
\hline $\mathrm{Cl}(1)-\operatorname{Zr}(1)-\mathrm{C}(7)$ & $114.28(6)$ \\
\hline$C l(2)-\operatorname{Zr}(1)-C(7)$ & $110.99(6)$ \\
\hline$C(32)-\operatorname{Zr}(1)-C(7)$ & $87.19(9)$ \\
\hline$C(31)-\operatorname{Zr}(1)-C(7)$ & $103.36(9)$ \\
\hline$C(30)-\operatorname{Zr}(1)-C(7)$ & $136.00(9)$ \\
\hline$C(33)-\operatorname{Zr}(1)-C(7)$ & $105.51(10)$ \\
\hline$C(34)-\operatorname{Zr}(1)-C(7)$ & $137.63(10)$ \\
\hline$N(4)-\operatorname{Zr}(2)-N(3)$ & $59.77(8)$ \\
\hline $\mathrm{N}(4)-\operatorname{Zr}(2)-\mathrm{Cl}(4)$ & $88.76(6)$ \\
\hline$N(3)-\operatorname{Zr}(2)-C 1(4)$ & $133.07(6)$ \\
\hline$N(4)-\operatorname{Zr}(2)-\operatorname{Cl}(3)$ & $133.55(6)$ \\
\hline$N(3)-\operatorname{Zr}(2)-C 1(3)$ & $88.28(6)$ \\
\hline $\operatorname{Cl}(4)-\operatorname{Zr}(2)-\operatorname{Cl}(3)$ & $91.18(3)$ \\
\hline
\end{tabular}




\begin{tabular}{|c|c|}
\hline$N(4)-\operatorname{Zr}(2)-C(37)$ & $136.85(13)$ \\
\hline$N(3)-\operatorname{Zr}(2)-C(37)$ & $133.66(11)$ \\
\hline $\operatorname{Cl}(4)-\operatorname{Zr}(2)-C(37)$ & $93.25(10)$ \\
\hline $\operatorname{Cl}(3)-\operatorname{Zr}(2)-C(37)$ & $89.53(12)$ \\
\hline$N(4)-\operatorname{Zr}(2)-C(36)$ & $130.91(11)$ \\
\hline$N(3)-\operatorname{Zr}(2)-C(36)$ & $100.90(11)$ \\
\hline $\operatorname{Cl}(4)-\operatorname{Zr}(2)-C(36)$ & $125.76(9)$ \\
\hline $\operatorname{Cl}(3)-\operatorname{Zr}(2)-C(36)$ & $84.54(10)$ \\
\hline$C(37)-\operatorname{Zr}(2)-C(36)$ & $32.91(13)$ \\
\hline$N(4)-\operatorname{Zr}(2)-C(38)$ & $105.12(13)$ \\
\hline$N(3)-\operatorname{Zr}(2)-C(38)$ & $134.29(11)$ \\
\hline $\operatorname{Cl}(4)-\operatorname{Zr}(2)-C(38)$ & $84.29(10)$ \\
\hline $\mathrm{Cl}(3)-\operatorname{Zr}(2)-\mathrm{C}(38)$ & $121.08(11)$ \\
\hline$C(37)-\operatorname{Zr}(2)-C(38)$ & $32.74(15)$ \\
\hline$C(36)-\operatorname{Zr}(2)-C(38)$ & $54.09(14)$ \\
\hline$N(4)-\operatorname{Zr}(2)-C(35)$ & $98.13(10)$ \\
\hline$N(3)-\operatorname{Zr}(2)-C(35)$ & $83.87(9)$ \\
\hline $\operatorname{Cl}(4)-\operatorname{Zr}(2)-C(35)$ & $138.17(8)$ \\
\hline $\operatorname{Cl}(3)-\operatorname{Zr}(2)-C(35)$ & $111.90(9)$ \\
\hline$C(37)-\operatorname{Zr}(2)-C(35)$ & $54.50(12)$ \\
\hline$C(36)-\operatorname{Zr}(2)-C(35)$ & $32.78(12)$ \\
\hline$C(38)-\operatorname{Zr}(2)-C(35)$ & $54.03(12)$ \\
\hline$N(4)-\operatorname{Zr}(2)-C(39)$ & $84.33(11)$ \\
\hline$N(3)-\operatorname{Zr}(2)-C(39)$ & $101.88(12)$ \\
\hline $\operatorname{Cl}(4)-\operatorname{Zr}(2)-C(39)$ & $109.03(10)$ \\
\hline $\operatorname{Cl}(3)-\operatorname{Zr}(2)-C(39)$ & $138.34(9)$ \\
\hline$C(37)-\operatorname{Zr}(2)-C(39)$ & $54.37(14)$ \\
\hline$C(36)-\operatorname{Zr}(2)-C(39)$ & $53.97(13)$ \\
\hline$C(38)-\operatorname{Zr}(2)-C(39)$ & $32.74(14)$ \\
\hline$C(35)-\operatorname{Zr}(2)-C(39)$ & $32.33(13)$ \\
\hline$N(4)-\operatorname{Zr}(2)-C(23)$ & $30.36(8)$ \\
\hline$N(3)-\operatorname{Zr}(2)-C(23)$ & $30.28(8)$ \\
\hline $\operatorname{Cl}(4)-\operatorname{Zr}(2)-C(23)$ & $108.91(6)$ \\
\hline $\operatorname{Cl}(3)-\operatorname{Zr}(2)-C(23)$ & $108.87(6)$ \\
\hline$C(37)-\operatorname{Zr}(2)-C(23)$ & $150.36(11)$ \\
\hline$C(36)-\operatorname{Zr}(2)-C(23)$ & $123.62(10)$ \\
\hline$C(38)-\operatorname{Zr}(2)-C(23)$ & $128.15(13)$ \\
\hline$C(35)-\operatorname{Zr}(2)-C(23)$ & $96.37(9)$ \\
\hline$C(39)-\operatorname{Zr}(2)-C(23)$ & $98.70(11)$ \\
\hline$C(13)-O(1)-C(17)$ & $118.13(19)$ \\
\hline$C(7)-N(1)-C(2)$ & $120.7(2)$ \\
\hline$C(7)-N(1)-\operatorname{Zr}(1)$ & $93.21(15)$ \\
\hline$C(2)-N(1)-\operatorname{Zr}(1)$ & $145.53(18)$ \\
\hline$C(7)-N(2)-C(5)$ & $121.3(2)$ \\
\hline$C(7)-N(2)-\operatorname{Zr}(1)$ & $93.23(16)$ \\
\hline$C(5)-N(2)-\operatorname{Zr}(1)$ & $143.39(17)$ \\
\hline$C(23)-N(3)-C(25)$ & $122.4(2)$ \\
\hline$C(23)-N(3)-\operatorname{Zr}(2)$ & $92.09(15)$ \\
\hline$C(25)-N(3)-\operatorname{Zr}(2)$ & $143.40(17)$ \\
\hline$C(23)-N(4)-C(28)$ & $124.0(2)$ \\
\hline$C(23)-N(4)-\operatorname{Zr}(2)$ & $92.36(15)$ \\
\hline $\mathrm{C}(28)-\mathrm{N}(4)-\operatorname{Zr}(2)$ & $139.76(16)$ \\
\hline$N(1)-C(2)-C(3)$ & $110.7(2)$ \\
\hline$N(1)-C(2)-C(1)$ & $111.5(2)$ \\
\hline$C(3)-C(2)-C(1)$ & $110.8(3)$ \\
\hline$N(2)-C(5)-C(4)$ & $110.6(2)$ \\
\hline$N(2)-C(5)-C(6)$ & $109.5(2)$ \\
\hline
\end{tabular}




\begin{tabular}{|c|c|}
\hline$C(4)-C(5)-C(6)$ & $112.5(2)$ \\
\hline $\mathrm{N}(1)-\mathrm{C}(7)-\mathrm{N}(2)$ & $113.3(2)$ \\
\hline$N(1)-C(7)-C(8)$ & $123.6(2)$ \\
\hline $\mathrm{N}(2)-\mathrm{C}(7)-\mathrm{C}(8)$ & $122.6(2)$ \\
\hline$N(1)-C(7)-\operatorname{Zr}(1)$ & $56.77(13)$ \\
\hline$N(2)-C(7)-\operatorname{Zr}(1)$ & $56.66(13)$ \\
\hline$C(8)-C(7)-\operatorname{Zr}(1)$ & $176.62(17)$ \\
\hline$C(9)-C(8)-C(13)$ & $118.7(2)$ \\
\hline$C(9)-C(8)-C(7)$ & $116.7(2)$ \\
\hline$C(13)-C(8)-C(7)$ & $124.6(2)$ \\
\hline$C(10)-C(9)-C(8)$ & $121.0(3)$ \\
\hline$C(11)-C(10)-C(9)$ & $119.4(2)$ \\
\hline$C(10)-C(11)-C(12)$ & $121.9(2)$ \\
\hline$C(13)-C(12)-C(11)$ & $117.8(2)$ \\
\hline$C(13)-C(12)-C(15)$ & $122.3(2)$ \\
\hline$C(11)-C(12)-C(15)$ & $119.9(2)$ \\
\hline$O(1)-C(13)-C(12)$ & $123.6(2)$ \\
\hline$O(1)-C(13)-C(8)$ & $115.2(2)$ \\
\hline$C(12)-C(13)-C(8)$ & $121.2(2)$ \\
\hline$C(18)-C(15)-C(12)$ & $110.18(19)$ \\
\hline $\mathrm{C}(18)-\mathrm{C}(15)-\mathrm{C}(14)$ & $109.8(2)$ \\
\hline$C(12)-C(15)-C(14)$ & $108.9(2)$ \\
\hline$C(18)-C(15)-C(16)$ & $109.2(2)$ \\
\hline$C(12)-C(15)-C(16)$ & $109.6(2)$ \\
\hline$C(14)-C(15)-C(16)$ & $109.1(2)$ \\
\hline$O(1)-C(17)-C(18)$ & $122.8(2)$ \\
\hline$O(1)-C(17)-C(22)$ & $116.0(2)$ \\
\hline$C(18)-C(17)-C(22)$ & $121.2(2)$ \\
\hline$C(19)-C(18)-C(17)$ & $118.3(2)$ \\
\hline$C(19)-C(18)-C(15)$ & $119.0(2)$ \\
\hline$C(17)-C(18)-C(15)$ & $122.7(2)$ \\
\hline$C(20)-C(19)-C(18)$ & $121.7(2)$ \\
\hline$C(19)-C(20)-C(21)$ & $119.1(2)$ \\
\hline$C(20)-C(21)-C(22)$ & $121.4(2)$ \\
\hline$C(21)-C(22)-C(17)$ & $118.2(2)$ \\
\hline$C(21)-C(22)-C(23)$ & $113.4(2)$ \\
\hline$C(17)-C(22)-C(23)$ & $128.1(2)$ \\
\hline$N(3)-C(23)-N(4)$ & $112.6(2)$ \\
\hline$N(3)-C(23)-C(22)$ & $124.4(2)$ \\
\hline $\mathrm{N}(4)-\mathrm{C}(23)-\mathrm{C}(22)$ & $121.6(2)$ \\
\hline$N(3)-C(23)-\operatorname{Zr}(2)$ & $57.63(13)$ \\
\hline$N(4)-C(23)-\operatorname{Zr}(2)$ & $57.28(12)$ \\
\hline$C(22)-C(23)-\operatorname{Zr}(2)$ & $154.57(17)$ \\
\hline$N(3)-C(25)-C(24)$ & $108.5(2)$ \\
\hline $\mathrm{N}(3)-\mathrm{C}(25)-\mathrm{C}(26)$ & $113.5(2)$ \\
\hline$C(24)-C(25)-C(26)$ & $109.0(2)$ \\
\hline $\mathrm{N}(4)-\mathrm{C}(28)-\mathrm{C}(29)$ & $108.3(2)$ \\
\hline $\mathrm{N}(4)-\mathrm{C}(28)-\mathrm{C}(27)$ & $114.0(2)$ \\
\hline$C(29)-C(28)-C(27)$ & $110.6(2)$ \\
\hline$C(34)-C(30)-C(31)$ & $108.2(3)$ \\
\hline$C(34)-C(30)-\operatorname{Zr}(1)$ & $74.16(19)$ \\
\hline$C(31)-C(30)-\operatorname{Zr}(1)$ & $73.40(17)$ \\
\hline$C(30)-C(31)-C(32)$ & $107.3(3)$ \\
\hline$C(30)-C(31)-\operatorname{Zr}(1)$ & $73.90(17)$ \\
\hline$C(32)-C(31)-\operatorname{Zr}(1)$ & $73.28(17)$ \\
\hline$C(33)-C(32)-C(31)$ & $108.2(3)$ \\
\hline$C(33)-C(32)-\operatorname{Zr}(1)$ & $74.39(17)$ \\
\hline
\end{tabular}




$\begin{array}{lc}C(31)-C(32)-\operatorname{Zr}(1) & 73.72(17) \\ C(32)-C(33)-C(34) & 108.1(3) \\ C(32)-C(33)-\operatorname{Zr}(1) & 73.13(17) \\ C(34)-C(33)-\operatorname{Zr}(1) & 74.01(19) \\ C(30)-C(34)-C(33) & 108.2(3) \\ C(30)-C(34)-\operatorname{Zr}(1) & 73.38(19) \\ C(33)-C(34)-\operatorname{Zr}(1) & 73.54(19) \\ C(39)-C(35)-C(36) & 107.9(3) \\ C(39)-C(35)-\operatorname{Zr}(2) & 74.0(2) \\ C(36)-C(35)-\operatorname{Zr}(2) & 72.97(19) \\ C(37)-C(36)-C(35) & 108.1(4) \\ C(37)-C(36)-\operatorname{Zr}(2) & 72.76(19) \\ C(35)-C(36)-\operatorname{Zr}(2) & 74.25(18) \\ C(38)-C(37)-C(36) & 107.8(3) \\ C(38)-C(37)-\operatorname{Zr}(2) & 74.65(19) \\ C(36)-C(37)-\operatorname{Zr}(2) & 74.33(19) \\ C(37)-C(38)-\operatorname{C}(39) & 108.1(3) \\ C(37)-C(38)-\operatorname{Zr}(2) & 72.61(19) \\ C(39)-C(38)-\operatorname{Zr}(2) & 74.17(18) \\ C(35)-C(39)-\operatorname{C}(38) & 108.1(3) \\ C(35)-C(39)-\operatorname{Zr}(2) & 73.71(19) \\ C(38)-C(39)-\operatorname{Zr}(2) & 73.1(2)\end{array}$


Figure S3. Molecular structure of 6 drawn with 50\% thermal ellipsoids.

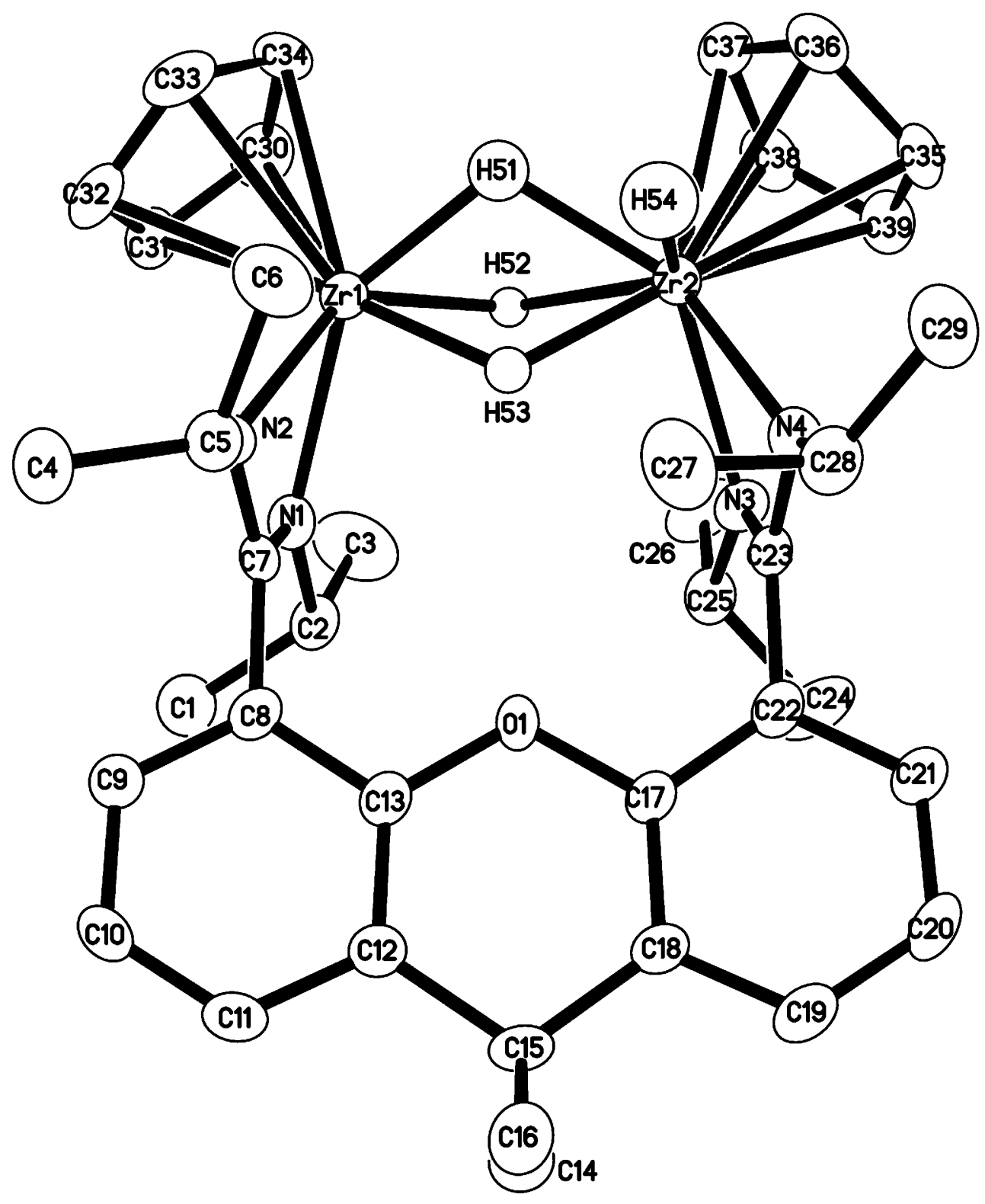


Table S11. Atomic Coordinates and $\mathrm{U}_{\mathrm{eq}}$ Values for 6

\begin{tabular}{|c|c|c|c|c|}
\hline & $\mathrm{x}$ & y & $\mathrm{z}$ & $\mathrm{U}(\mathrm{eq})$ \\
\hline $\operatorname{Zr}(1)$ & $-2427(1)$ & $-6755(1)$ & $-2950(1)$ & $22(1)$ \\
\hline $\operatorname{Zr}(2)$ & $-1472(1)$ & $-5614(1)$ & $-1660(1)$ & $24(1)$ \\
\hline$O(1)$ & $-1020(1)$ & $-8709(2)$ & $-1933(3)$ & $25(1)$ \\
\hline $\mathrm{N}(1)$ & $-1992(2)$ & $-8015(3)$ & $-3836(3)$ & $25(1)$ \\
\hline $\mathrm{N}(2)$ & $-2554(2)$ & $-8254(3)$ & $-2314(3)$ & $23(1)$ \\
\hline $\mathrm{N}(3)$ & $-648(2)$ & $-6409(3)$ & $-2210(3)$ & $26(1)$ \\
\hline $\mathrm{N}(4)$ & $-919(2)$ & $-6455(3)$ & $-390(3)$ & $27(1)$ \\
\hline$C(1)$ & $-1748(2)$ & $-9136(4)$ & $-5441(5)$ & $38(1)$ \\
\hline$C(2)$ & $-1565(2)$ & $-8254(4)$ & $-4730(4)$ & $25(1)$ \\
\hline$C(3)$ & $-1497(3)$ & $-7394(4)$ & $-5502(5)$ & $51(2)$ \\
\hline$C(4)$ & $-3180(2)$ & $-9415(4)$ & $-1289(5)$ & $36(1)$ \\
\hline$C(5)$ & $-2718(2)$ & $-8649(4)$ & $-1188(4)$ & $25(1)$ \\
\hline$C(6)$ & $-2940(2)$ & $-7806(4)$ & $-460(4)$ & $37(1)$ \\
\hline$C(7)$ & $-2122(2)$ & $-8598(3)$ & $-2960(5)$ & $21(1)$ \\
\hline$C(8)$ & $-1825(2)$ & $-9536(3)$ & $-2718(4)$ & $23(1)$ \\
\hline$C(9)$ & $-2104(2)$ & $-10388(3)$ & $-3018(5)$ & $27(1)$ \\
\hline$C(10)$ & $-1836(2)$ & $-11279(3)$ & $-2825(5)$ & $28(1)$ \\
\hline$C(11)$ & $-1293(2)$ & $-11315(4)$ & $-2351(4)$ & $28(1)$ \\
\hline$C(12)$ & $-998(2)$ & $-10478(3)$ & $-2046(4)$ & $25(1)$ \\
\hline$C(13)$ & $-1267(2)$ & $-9592(3)$ & $-2217(4)$ & $24(1)$ \\
\hline$C(14)$ & $26(2)$ & $-10967(4)$ & $-2447(5)$ & $41(1)$ \\
\hline$C(15)$ & $-386(2)$ & $-10546(3)$ & $-1539(5)$ & $27(1)$ \\
\hline$C(16)$ & $-405(2)$ & $-11227(4)$ & $-487(5)$ & $41(2)$ \\
\hline$C(17)$ & $-489(2)$ & $-8715(3)$ & $-1375(4)$ & $21(1)$ \\
\hline$C(18)$ & $-177(2)$ & $-9552(3)$ & $-1173(4)$ & $23(1)$ \\
\hline$C(19)$ & $354(2)$ & $-9457(4)$ & $-608(4)$ & $30(1)$ \\
\hline$C(20)$ & $562(2)$ & $-8566(4)$ & $-253(5)$ & $33(1)$ \\
\hline$C(21)$ & $240(2)$ & $-7746(4)$ & $-453(4)$ & $27(1)$ \\
\hline$C(22)$ & $-292(2)$ & $-7802(4)$ & $-1025(4)$ & $24(1)$ \\
\hline$C(23)$ & $-618(2)$ & $-6888(3)$ & $-1216(4)$ & $21(1)$ \\
\hline C (24) & $269(2)$ & $-6935(5)$ & $-3168(5)$ & $48(2)$ \\
\hline$C(25)$ & $-373(2)$ & $-6718(4)$ & $-3276(4)$ & $28(1)$ \\
\hline$C(26)$ & $-447(2)$ & $-5925(4)$ & $-4174(5)$ & $42(2)$ \\
\hline$C(27)$ & $-1469(2)$ & $-7558(4)$ & $879(5)$ & $44(2)$ \\
\hline$C(28)$ & $-962(2)$ & $-6869(4)$ & $776(4)$ & $31(1)$ \\
\hline$C(29)$ & $-1013(2)$ & $-6029(4)$ & $1624(4)$ & 41 (1) \\
\hline$C(30)$ & $-2890(2)$ & $-5770(4)$ & $-4523(5)$ & $38(1)$ \\
\hline$C(31)$ & $-3061(2)$ & $-6723(4)$ & $-4707(4)$ & $33(1)$ \\
\hline$C(32)$ & $-3413(2)$ & $-7016(4)$ & $-3800(5)$ & $38(1)$ \\
\hline$C(33)$ & $-3461(2)$ & $-6230(4)$ & $-3043(6)$ & $43(1)$ \\
\hline$C(34)$ & $-3135(2)$ & $-5456(4)$ & $-3501(5)$ & $43(2)$ \\
\hline$C(35)$ & $-1019(2)$ & $-4111(4)$ & $-800(5)$ & $38(1)$ \\
\hline$C(36)$ & $-1621(3)$ & $-3934(4)$ & $-858(5)$ & $40(2)$ \\
\hline$C(37)$ & $-1767(2)$ & $-3887(4)$ & $-2024(5)$ & $33(1)$ \\
\hline$C(38)$ & $-1265(2)$ & $-4024(4)$ & $-2678(5)$ & $38(2)$ \\
\hline C (39) & $-802(2)$ & $-4156(4)$ & $-1910(5)$ & $38(2)$ \\
\hline
\end{tabular}


Table S12. Calculated and refined coordinates of H-atoms for $\mathbf{6}$

\begin{tabular}{|c|c|c|c|c|}
\hline & $x$ & $\mathrm{y}$ & z & $\mathrm{U}(\mathrm{eq})$ \\
\hline $\mathrm{H}(1 \mathrm{~A})$ & -1736 & -9718 & -4958 & 57 \\
\hline $\mathrm{H}(1 \mathrm{~B})$ & -1484 & -9216 & -6091 & 57 \\
\hline $\mathrm{H}(1 \mathrm{C})$ & -2141 & -9039 & -5728 & 57 \\
\hline $\mathrm{H}(2)$ & -1186 & -8395 & -4355 & 31 \\
\hline $\mathrm{H}(3 \mathrm{~A})$ & -1853 & -7296 & -5940 & 76 \\
\hline $\mathrm{H}(3 \mathrm{~B})$ & -1176 & -7506 & -6032 & 76 \\
\hline $\mathrm{H}(3 \mathrm{C})$ & -1418 & -6816 & -5037 & 76 \\
\hline $\mathrm{H}(4 \mathrm{~A})$ & -3511 & -9150 & -1708 & 54 \\
\hline $\mathrm{H}(4 \mathrm{~B})$ & -3303 & -9617 & -520 & 54 \\
\hline $\mathrm{H}(4 \mathrm{C})$ & -3027 & -9976 & -1705 & 54 \\
\hline H (5) & -2370 & -8936 & -811 & 30 \\
\hline$H(6 A)$ & -2651 & -7288 & -445 & 56 \\
\hline $\mathrm{H}(6 \mathrm{~B})$ & -3013 & -8032 & 325 & 56 \\
\hline $\mathrm{H}(6 \mathrm{C})$ & -3299 & -7557 & -793 & 56 \\
\hline H (9) & -2477 & -10366 & -3354 & 33 \\
\hline $\mathrm{H}(10)$ & -2029 & -11862 & -3023 & 34 \\
\hline $\mathrm{H}(11)$ & -1116 & -11928 & -2230 & 33 \\
\hline $\mathrm{H}(14 \mathrm{~A})$ & 48 & -10524 & -3104 & 61 \\
\hline $\mathrm{H}(14 \mathrm{~B})$ & -119 & -11598 & -2705 & 61 \\
\hline $\mathrm{H}(14 \mathrm{C})$ & 411 & -11047 & -2111 & 61 \\
\hline $\mathrm{H}(16 \mathrm{~A})$ & -17 & -11285 & -159 & 61 \\
\hline $\mathrm{H}(16 \mathrm{~B})$ & -541 & -11868 & -726 & 61 \\
\hline $\mathrm{H}(16 \mathrm{C})$ & -667 & -10959 & 91 & 61 \\
\hline H (19) & 578 & -10021 & -465 & 36 \\
\hline $\mathrm{H}(20)$ & 923 & -8521 & 127 & 39 \\
\hline $\mathrm{H}(21)$ & 380 & -7134 & -202 & 33 \\
\hline $\mathrm{H}(24 \mathrm{~A})$ & 461 & -6397 & -2774 & 71 \\
\hline $\mathrm{H}(24 \mathrm{~B})$ & 436 & -7017 & -3935 & 71 \\
\hline $\mathrm{H}(24 \mathrm{C})$ & 322 & -7533 & -2725 & 71 \\
\hline H (25) & -573 & -7315 & -3556 & 34 \\
\hline $\mathrm{H}(26 \mathrm{~A})$ & -854 & -5738 & -4219 & 63 \\
\hline $\mathrm{H}(26 \mathrm{~B})$ & -319 & -6167 & -4924 & 63 \\
\hline $\mathrm{H}(26 \mathrm{C})$ & -214 & -5360 & -3959 & 63 \\
\hline $\mathrm{H}(27 \mathrm{~A})$ & -1828 & -7205 & 727 & 65 \\
\hline $\mathrm{H}(27 \mathrm{~B})$ & -1481 & -7829 & 1657 & 65 \\
\hline $\mathrm{H}(27 \mathrm{C})$ & -1427 & -8085 & 320 & 65 \\
\hline $\mathrm{H}(28)$ & -600 & -7235 & 945 & 37 \\
\hline $\mathrm{H}(29 \mathrm{~A})$ & -676 & -5604 & 1547 & 61 \\
\hline $\mathrm{H}(29 \mathrm{~B})$ & -1031 & -6284 & 2409 & 61 \\
\hline $\mathrm{H}(29 \mathrm{C})$ & -1364 & -5659 & 1461 & 61 \\
\hline $\mathrm{H}(30)$ & -2647 & -5398 & -5011 & 53 \\
\hline H (31) & -2956 & -7112 & -5347 & 46 \\
\hline $\mathrm{H}(32)$ & -3588 & -7635 & -3711 & 53 \\
\hline H (33) & -3674 & -6220 & -2347 & 59 \\
\hline H (34) & -3091 & -4832 & -3168 & 60 \\
\hline H (35) & -800 & -4186 & -116 & 53 \\
\hline $\mathrm{H}(36)$ & -1877 & -3861 & -226 & 56 \\
\hline $\mathrm{H}(37)$ & -2143 & -3781 & -2322 & 46 \\
\hline $\mathrm{H}(38)$ & -1241 & -4027 & -3492 & 52 \\
\hline H (39) & -410 & -4258 & -2115 & 54 \\
\hline
\end{tabular}




\begin{tabular}{lllll}
$\mathrm{H}(51)$ & $-2283(19)$ & $-5660(30)$ & $-2050(40)$ & $32(14)$ \\
$\mathrm{H}(52)$ & $-1790(16)$ & $-5960(30)$ & $-3250(30)$ & $15(11)$ \\
$\mathrm{H}(53)$ & $-1831(16)$ & $-6820(30)$ & $-1860(40)$ & $18(11)$ \\
$\mathrm{H}(54)$ & $-1880(20)$ & $-5740(40)$ & $-450(50)$ & $49(16)$ \\
\hline
\end{tabular}


Table S13. Anisotropic thermal parameters for 6

\begin{tabular}{|c|c|c|c|c|c|c|}
\hline & $\mathrm{U} 11$ & $\mathrm{U} 22$ & U33 & U23 & U13 & $\mathrm{U} 12$ \\
\hline$\overline{\operatorname{Zr}(1)}$ & $21(1)$ & $20(1)$ & $26(1)$ & $1(1)$ & $-3(1)$ & $1(1)$ \\
\hline $\operatorname{Zr}(2)$ & $22(1)$ & $20(1)$ & $29(1)$ & $-1(1)$ & $-2(1)$ & $-1(1)$ \\
\hline$O(1)$ & $20(2)$ & $22(2)$ & $32(2)$ & $0(1)$ & $-6(1)$ & $-5(1)$ \\
\hline $\mathrm{N}(1)$ & $24(2)$ & $24(2)$ & $26(2)$ & $3(2)$ & $-2(2)$ & $-4(2)$ \\
\hline $\mathrm{N}(2)$ & $22(2)$ & $21(2)$ & $26(2)$ & $0(2)$ & $4(2)$ & $-2(2)$ \\
\hline N (3) & $26(2)$ & $28(2)$ & $25(2)$ & $4(2)$ & $-4(2)$ & $3(2)$ \\
\hline N (4) & $27(2)$ & $27(2)$ & $27(2)$ & $0(2)$ & $2(2)$ & $-6(2)$ \\
\hline$C(1)$ & $33(3)$ & $44(3)$ & $37(3)$ & $-12(3)$ & $6(3)$ & $0(3)$ \\
\hline$C(2)$ & $20(2)$ & $34(3)$ & $22(3)$ & $-3(2)$ & $4(2)$ & $-2(2)$ \\
\hline C (3) & $74(4)$ & $46(4)$ & $33(3)$ & $1(3)$ & $26(3)$ & $1(3)$ \\
\hline$C(4)$ & $31(3)$ & $34(3)$ & $43(3)$ & $1(3)$ & $3(2)$ & $-9(3)$ \\
\hline$C(5)$ & $26(3)$ & $27(3)$ & $22(2)$ & $1(2)$ & $0(2)$ & $-1(2)$ \\
\hline$C(6)$ & $51(3)$ & $36(3)$ & $26(3)$ & $-2(2)$ & $10(3)$ & $-2(3)$ \\
\hline$C(7)$ & $18(2)$ & $17(2)$ & $27(2)$ & $-4(3)$ & $-2(3)$ & $-3(2)$ \\
\hline$C(8)$ & $20(2)$ & $27(3)$ & $23(3)$ & $4(2)$ & $-2(2)$ & $2(2)$ \\
\hline$C(9)$ & $24(2)$ & $26(2)$ & $32(3)$ & $-1(3)$ & $-1(3)$ & $1(2)$ \\
\hline$C(10)$ & $32(2)$ & $20(2)$ & $33(3)$ & $-5(3)$ & $0(3)$ & $-2(2)$ \\
\hline C (11) & $29(3)$ & $22(3)$ & $32(3)$ & $0(2)$ & $7(2)$ & $5(2)$ \\
\hline C (12) & $27(2)$ & $25(3)$ & $23(3)$ & $1(2)$ & $-1(2)$ & $3(2)$ \\
\hline C (13) & $22(2)$ & $29(3)$ & $20(2)$ & $-1(2)$ & $-3(2)$ & $2(2)$ \\
\hline$C(14)$ & $34(3)$ & $43(3)$ & $45(3)$ & $-15(3)$ & $-1(3)$ & $8(3)$ \\
\hline C (15) & $25(2)$ & $24(2)$ & $33(3)$ & $4(2)$ & $3(2)$ & $7(2)$ \\
\hline$C(16)$ & $42(3)$ & $33(3)$ & $48(4)$ & $14(3)$ & $-18(3)$ & $-5(3)$ \\
\hline C (17) & $19(2)$ & $25(2)$ & $20(3)$ & $4(2)$ & $-2(2)$ & $1(2)$ \\
\hline C (18) & $22(2)$ & $24(3)$ & $25(2)$ & $5(2)$ & $1(2)$ & $3(2)$ \\
\hline C (19) & $21(2)$ & $38(3)$ & $31(3)$ & $4(3)$ & $2(2)$ & $5(2)$ \\
\hline C (20) & $19(2)$ & $42(3)$ & $38(3)$ & $5(3)$ & $-9(2)$ & $-3(2)$ \\
\hline$C(21)$ & $25(2)$ & $34(3)$ & $23(3)$ & $2(2)$ & $-5(2)$ & $4(2)$ \\
\hline C (22) & $20(2)$ & $28(3)$ & $23(3)$ & $5(2)$ & $-1(2)$ & $-2(2)$ \\
\hline C (23) & $15(2)$ & $23(3)$ & $25(3)$ & $-3(2)$ & $-1(2)$ & $-3(2)$ \\
\hline C (24) & $32(3)$ & $66(4)$ & $44(4)$ & $16(3)$ & $2(3)$ & $17(3)$ \\
\hline C (25) & $24(2)$ & $32(3)$ & $28(3)$ & $0(2)$ & $3(2)$ & $-4(2)$ \\
\hline$C(26)$ & $32(3)$ & $64(4)$ & $30(3)$ & $15(3)$ & $7(2)$ & $12(3)$ \\
\hline$C(27)$ & $57(4)$ & $45(3)$ & $29(3)$ & $1(3)$ & $3(3)$ & $-13(3)$ \\
\hline C (28) & $32(3)$ & $37(3)$ & $24(3)$ & $3(2)$ & $-3(2)$ & $0(3)$ \\
\hline C (29) & $50(3)$ & $46(3)$ & $28(3)$ & $-4(3)$ & $-3(3)$ & $-5(3)$ \\
\hline$C(30)$ & $40(3)$ & $31(3)$ & $42(4)$ & $19(3)$ & $-13(3)$ & $2(3)$ \\
\hline C (31) & $33(3)$ & $37(3)$ & $30(3)$ & $-1(3)$ & $-18(2)$ & $2(3)$ \\
\hline C (32) & $20(3)$ & $37(3)$ & $56(4)$ & $14(3)$ & $-11(3)$ & $0(2)$ \\
\hline C (33) & $28(3)$ & $54(4)$ & $45(3)$ & $-3(3)$ & $-7(3)$ & $18(3)$ \\
\hline C (34) & $44(3)$ & $19(3)$ & $67(4)$ & $-9(3)$ & $-31(3)$ & $16(3)$ \\
\hline C (35) & $37(3)$ & $21(3)$ & $55(4)$ & $-8(3)$ & $-15(3)$ & $-8(3)$ \\
\hline$C(36)$ & $45(3)$ & $21(3)$ & $53(4)$ & $-8(3)$ & $-8(3)$ & $1(3)$ \\
\hline C (37) & $27(3)$ & $21(3)$ & $51(4)$ & $5(2)$ & $-8(2)$ & $0(2)$ \\
\hline C (38) & $45(3)$ & $21(3)$ & $47(4)$ & $6(3)$ & $-2(3)$ & $-10(3)$ \\
\hline C (39) & $30(3)$ & $27(3)$ & $59(5)$ & $0(3)$ & $0(3)$ & $-11(2)$ \\
\hline
\end{tabular}


Table S14. Bond lengths for 6

\begin{tabular}{|c|c|}
\hline $\operatorname{Zr}(1)-N(2)$ & $2.216(4)$ \\
\hline $\operatorname{Zr}(1)-\mathrm{N}(1)$ & $2.259(4)$ \\
\hline $\operatorname{Zr}(1)-C(33)$ & $2.504(4)$ \\
\hline $\operatorname{Zr}(1)-C(34)$ & $2.512(5)$ \\
\hline $\operatorname{Zr}(1)-C(32)$ & $2.515(5)$ \\
\hline $\operatorname{Zr}(1)-C(31)$ & $2.519(5)$ \\
\hline $\operatorname{Zr}(1)-C(30)$ & $2.519(5)$ \\
\hline $\operatorname{Zr}(1)-C(7)$ & $2.639(4)$ \\
\hline $\operatorname{Zr}(1)-\operatorname{Zr}(2)$ & $3.1050(6)$ \\
\hline $\operatorname{Zr}(1)-\mathrm{H}(51)$ & $1.86(5)$ \\
\hline $\operatorname{Zr}(1)-\mathrm{H}(52)$ & $1.88(4)$ \\
\hline $\operatorname{Zr}(1)-\mathrm{H}(53)$ & $1.88(4)$ \\
\hline $\operatorname{Zr}(2)-N(4)$ & $2.273(4)$ \\
\hline $\operatorname{Zr}(2)-N(3)$ & $2.293(4)$ \\
\hline $\operatorname{Zr}(2)-C(37)$ & $2.514(5)$ \\
\hline $\operatorname{Zr}(2)-C(36)$ & $2.522(6)$ \\
\hline $\operatorname{Zr}(2)-C(35)$ & $2.531(5)$ \\
\hline $\operatorname{Zr}(2)-C(38)$ & $2.539(5)$ \\
\hline $\operatorname{Zr}(2)-C(39)$ & $2.557(5)$ \\
\hline $\operatorname{Zr}(2)-C(23)$ & $2.695(5)$ \\
\hline $\mathrm{Zr}(2)-\mathrm{H}(51)$ & $1.94(4)$ \\
\hline $\operatorname{Zr}(2)-\mathrm{H}(52)$ & $2.05(4)$ \\
\hline $\operatorname{Zr}(2)-\mathrm{H}(53)$ & $1.87(4)$ \\
\hline $\mathrm{Zr}(2)-\mathrm{H}(54)$ & $1.71(6)$ \\
\hline$O(1)-C(13)$ & $1.387(5)$ \\
\hline $\mathrm{O}(1)-\mathrm{C}(17)$ & $1.392(5)$ \\
\hline $\mathrm{N}(1)-\mathrm{C}(7)$ & $1.334(6)$ \\
\hline$N(1)-C(2)$ & $1.474(6)$ \\
\hline$N(2)-C(7)$ & $1.339(6)$ \\
\hline$N(2)-C(5)$ & $1.470(6)$ \\
\hline$N(3)-C(23)$ & $1.334(6)$ \\
\hline$N(3)-C(25)$ & $1.459(6)$ \\
\hline$N(4)-C(23)$ & $1.330(6)$ \\
\hline$N(4)-C(28)$ & $1.477(6)$ \\
\hline$C(1)-C(2)$ & $1.532(7)$ \\
\hline $\mathrm{C}(1)-\mathrm{H}(1 \mathrm{~A})$ & 0.9800 \\
\hline $\mathrm{C}(1)-\mathrm{H}(1 \mathrm{~B})$ & 0.9800 \\
\hline $\mathrm{C}(1)-\mathrm{H}(1 \mathrm{C})$ & 0.9800 \\
\hline$C(2)-C(3)$ & $1.496(7)$ \\
\hline $\mathrm{C}(2)-\mathrm{H}(2)$ & 1.0000 \\
\hline$C(3)-H(3 A)$ & 0.9800 \\
\hline$C(3)-H(3 B)$ & 0.9800 \\
\hline $\mathrm{C}(3)-\mathrm{H}(3 \mathrm{C})$ & 0.9800 \\
\hline$C(4)-C(5)$ & $1.510(7)$ \\
\hline$C(4)-H(4 A)$ & 0.9800 \\
\hline$C(4)-H(4 B)$ & 0.9800 \\
\hline $\mathrm{C}(4)-\mathrm{H}(4 \mathrm{C})$ & 0.9800 \\
\hline$C(5)-C(6)$ & $1.528(7)$ \\
\hline $\mathrm{C}(5)-\mathrm{H}(5)$ & 1.0000 \\
\hline$C(6)-H(6 A)$ & 0.9800 \\
\hline$C(6)-H(6 B)$ & 0.9800 \\
\hline $\mathrm{C}(6)-\mathrm{H}(6 \mathrm{C})$ & 0.9800 \\
\hline
\end{tabular}




\begin{tabular}{|c|c|}
\hline$C(7)-C(8)$ & $1.492(6)$ \\
\hline$C(8)-C(9)$ & $1.387(6)$ \\
\hline$C(8)-C(13)$ & $1.420(6)$ \\
\hline$C(9)-C(10)$ & $1.394(6)$ \\
\hline $\mathrm{C}(9)-\mathrm{H}(9)$ & 0.9500 \\
\hline$C(10)-C(11)$ & $1.376(7)$ \\
\hline $\mathrm{C}(10)-\mathrm{H}(10)$ & 0.9500 \\
\hline$C(11)-C(12)$ & $1.388(7)$ \\
\hline $\mathrm{C}(11)-\mathrm{H}(11)$ & 0.9500 \\
\hline$C(12)-C(13)$ & $1.387(6)$ \\
\hline$C(12)-C(15)$ & $1.540(6)$ \\
\hline$C(14)-C(15)$ & $1.538(7)$ \\
\hline $\mathrm{C}(14)-\mathrm{H}(14 \mathrm{~A})$ & 0.9800 \\
\hline $\mathrm{C}(14)-\mathrm{H}(14 \mathrm{~B})$ & 0.9800 \\
\hline $\mathrm{C}(14)-\mathrm{H}(14 \mathrm{C})$ & 0.9800 \\
\hline$C(15)-C(18)$ & $1.514(6)$ \\
\hline$C(15)-C(16)$ & $1.544(7)$ \\
\hline $\mathrm{C}(16)-\mathrm{H}(16 \mathrm{~A})$ & 0.9800 \\
\hline $\mathrm{C}(16)-\mathrm{H}(16 \mathrm{~B})$ & 0.9800 \\
\hline $\mathrm{C}(16)-\mathrm{H}(16 \mathrm{C})$ & 0.9800 \\
\hline$C(17)-C(18)$ & $1.382(6)$ \\
\hline$C(17)-C(22)$ & $1.400(6)$ \\
\hline$C(18)-C(19)$ & $1.403(6)$ \\
\hline$C(19)-C(20)$ & $1.383(7)$ \\
\hline $\mathrm{C}(19)-\mathrm{H}(19)$ & 0.9500 \\
\hline$C(20)-C(21)$ & $1.374(7)$ \\
\hline $\mathrm{C}(20)-\mathrm{H}(20)$ & 0.9500 \\
\hline$C(21)-C(22)$ & $1.404(6)$ \\
\hline $\mathrm{C}(21)-\mathrm{H}(21)$ & 0.9500 \\
\hline$C(22)-C(23)$ & $1.487(7)$ \\
\hline$C(24)-C(25)$ & $1.524(6)$ \\
\hline $\mathrm{C}(24)-\mathrm{H}(24 \mathrm{~A})$ & 0.9800 \\
\hline $\mathrm{C}(24)-\mathrm{H}(24 \mathrm{~B})$ & 0.9800 \\
\hline $\mathrm{C}(24)-\mathrm{H}(24 \mathrm{C})$ & 0.9800 \\
\hline$C(25)-C(26)$ & $1.522(7)$ \\
\hline $\mathrm{C}(25)-\mathrm{H}(25)$ & 1.0000 \\
\hline $\mathrm{C}(26)-\mathrm{H}(26 \mathrm{~A})$ & 0.9800 \\
\hline $\mathrm{C}(26)-\mathrm{H}(26 \mathrm{~B})$ & 0.9800 \\
\hline $\mathrm{C}(26)-\mathrm{H}(26 \mathrm{C})$ & 0.9800 \\
\hline$C(27)-C(28)$ & $1.516(7)$ \\
\hline $\mathrm{C}(27)-\mathrm{H}(27 \mathrm{~A})$ & 0.9800 \\
\hline $\mathrm{C}(27)-\mathrm{H}(27 \mathrm{~B})$ & 0.9800 \\
\hline $\mathrm{C}(27)-\mathrm{H}(27 \mathrm{C})$ & 0.9800 \\
\hline$C(28)-C(29)$ & $1.527(7)$ \\
\hline $\mathrm{C}(28)-\mathrm{H}(28)$ & 1.0000 \\
\hline $\mathrm{C}(29)-\mathrm{H}(29 A)$ & 0.9800 \\
\hline $\mathrm{C}(29)-\mathrm{H}(29 \mathrm{~B})$ & 0.9800 \\
\hline $\mathrm{C}(29)-\mathrm{H}(29 \mathrm{C})$ & 0.9800 \\
\hline$C(30)-C(34)$ & $1.387(8)$ \\
\hline$C(30)-C(31)$ & $1.390(7)$ \\
\hline $\mathrm{C}(30)-\mathrm{H}(30)$ & 0.9500 \\
\hline$C(31)-C(32)$ & $1.395(7)$ \\
\hline $\mathrm{C}(31)-\mathrm{H}(31)$ & 0.9500 \\
\hline$C(32)-C(33)$ & $1.401(8)$ \\
\hline $\mathrm{C}(32)-\mathrm{H}(32)$ & 0.9500 \\
\hline$C(33)-C(34)$ & $1.412(8)$ \\
\hline $\mathrm{C}(33)-\mathrm{H}(33)$ & 0.9500 \\
\hline
\end{tabular}




$\begin{array}{ll}\mathrm{C}(34)-\mathrm{H}(34) & 0.9500 \\ \mathrm{C}(35)-\mathrm{C}(39) & 1.387(8) \\ \mathrm{C}(35)-\mathrm{C}(36) & 1.419(8) \\ \mathrm{C}(35)-\mathrm{H}(35) & 0.9500 \\ \mathrm{C}(36)-\mathrm{C}(37) & 1.400(8) \\ \mathrm{C}(36)-\mathrm{H}(36) & 0.9500 \\ \mathrm{C}(37)-\mathrm{C}(38) & 1.404(7) \\ \mathrm{C}(37)-\mathrm{H}(37) & 0.9500 \\ \mathrm{C}(38)-\mathrm{C}(39) & 1.408(7) \\ \mathrm{C}(38)-\mathrm{H}(38) & 0.9500 \\ \mathrm{C}(39)-\mathrm{H}(39) & 0.9500\end{array}$


Table S15. Bond angles for 6

\begin{tabular}{|c|c|}
\hline $\mathrm{N}(2)-\mathrm{Zr}(1)-\mathrm{N}(1)$ & $59.60(14)$ \\
\hline$N(2)-\operatorname{Zr}(1)-C(33)$ & $99.05(18)$ \\
\hline$N(1)-\operatorname{Zr}(1)-C(33)$ & $129.04(17)$ \\
\hline$N(2)-\operatorname{Zr}(1)-C(34)$ & $131.61(17)$ \\
\hline $\mathrm{N}(1)-\operatorname{Zr}(1)-\mathrm{C}(34)$ & $136.37(17)$ \\
\hline$C(33)-\operatorname{Zr}(1)-C(34)$ & $32.69(19)$ \\
\hline $\mathrm{N}(2)-\operatorname{Zr}(1)-\mathrm{C}(32)$ & $82.95(16)$ \\
\hline $\mathrm{N}(1)-\operatorname{Zr}(1)-\mathrm{C}(32)$ & $96.66(17)$ \\
\hline$C(33)-\operatorname{Zr}(1)-C(32)$ & $32.42(18)$ \\
\hline$C(34)-\operatorname{Zr}(1)-C(32)$ & $53.76(18)$ \\
\hline$N(2)-\operatorname{Zr}(1)-C(31)$ & $102.15(16)$ \\
\hline $\mathrm{N}(1)-\operatorname{Zr}(1)-\mathrm{C}(31)$ & $84.45(16)$ \\
\hline$C(33)-\operatorname{Zr}(1)-C(31)$ & $53.3(2)$ \\
\hline$C(34)-\operatorname{Zr}(1)-C(31)$ & $53.09(18)$ \\
\hline$C(32)-\operatorname{Zr}(1)-C(31)$ & $32.17(17)$ \\
\hline$N(2)-\operatorname{Zr}(1)-C(30)$ & $133.62(16)$ \\
\hline$N(1)-\operatorname{Zr}(1)-C(30)$ & $105.86(17)$ \\
\hline$C(33)-\operatorname{Zr}(1)-C(30)$ & $53.5(2)$ \\
\hline$C(34)-\operatorname{Zr}(1)-C(30)$ & $32.01(18)$ \\
\hline$C(32)-\operatorname{Zr}(1)-C(30)$ & $53.50(18)$ \\
\hline$C(31)-\operatorname{Zr}(1)-C(30)$ & $32.04(16)$ \\
\hline$N(2)-\operatorname{Zr}(1)-C(7)$ & $30.45(14)$ \\
\hline$N(1)-\operatorname{Zr}(1)-C(7)$ & $30.36(15)$ \\
\hline$C(33)-\operatorname{Zr}(1)-C(7)$ & $122.30(16)$ \\
\hline$C(34)-\operatorname{Zr}(1)-C(7)$ & $149.40(16)$ \\
\hline$C(32)-\operatorname{Zr}(1)-C(7)$ & $95.94(16)$ \\
\hline$C(31)-\operatorname{Zr}(1)-C(7)$ & $99.76(17)$ \\
\hline$C(30)-\operatorname{Zr}(1)-C(7)$ & $129.09(19)$ \\
\hline$N(2)-\operatorname{Zr}(1)-\operatorname{Zr}(2)$ & $113.91(10)$ \\
\hline$N(1)-\operatorname{Zr}(1)-\operatorname{Zr}(2)$ & $106.96(10)$ \\
\hline$C(33)-\operatorname{Zr}(1)-\operatorname{Zr}(2)$ & $123.85(14)$ \\
\hline$C(34)-\operatorname{Zr}(1)-\operatorname{Zr}(2)$ & $103.21(13)$ \\
\hline$C(32)-\operatorname{Zr}(1)-\operatorname{Zr}(2)$ & $155.65(14)$ \\
\hline$C(31)-\operatorname{Zr}(1)-\operatorname{Zr}(2)$ & $143.17(13)$ \\
\hline$C(30)-\operatorname{Zr}(1)-\operatorname{Zr}(2)$ & $112.47(13)$ \\
\hline$C(7)-\operatorname{Zr}(1)-\operatorname{Zr}(2)$ & $107.39(10)$ \\
\hline$N(2)-\operatorname{Zr}(1)-H(51)$ & $126.2(14)$ \\
\hline$N(1)-\operatorname{Zr}(1)-\mathrm{H}(51)$ & $142.9(14)$ \\
\hline $\mathrm{C}(33)-\operatorname{Zr}(1)-\mathrm{H}(51)$ & $87.9(14)$ \\
\hline $\mathrm{C}(34)-\operatorname{Zr}(1)-\mathrm{H}(51)$ & $71.6(14)$ \\
\hline $\mathrm{C}(32)-\operatorname{Zr}(1)-\mathrm{H}(51)$ & $120.0(14)$ \\
\hline $\mathrm{C}(31)-\operatorname{Zr}(1)-\mathrm{H}(51)$ & $123.1(14)$ \\
\hline $\mathrm{C}(30)-\operatorname{Zr}(1)-\mathrm{H}(51)$ & $92.8(14)$ \\
\hline $\mathrm{C}(7)-\operatorname{Zr}(1)-\mathrm{H}(51)$ & $137.1(14)$ \\
\hline $\operatorname{Zr}(2)-\operatorname{Zr}(1)-\mathrm{H}(51)$ & $36.0(14)$ \\
\hline $\mathrm{N}(2)-\operatorname{Zr}(1)-\mathrm{H}(52)$ & $135.6(12)$ \\
\hline$N(1)-\operatorname{Zr}(1)-H(52)$ & $90.8(12)$ \\
\hline $\mathrm{C}(33)-\operatorname{Zr}(1)-\mathrm{H}(52)$ & $125.2(12)$ \\
\hline $\mathrm{C}(34)-\operatorname{Zr}(1)-\mathrm{H}(52)$ & $92.8(12)$ \\
\hline $\mathrm{C}(32)-\operatorname{Zr}(1)-\mathrm{H}(52)$ & $136.5(12)$ \\
\hline $\mathrm{C}(31)-\operatorname{Zr}(1)-\mathrm{H}(52)$ & $107.3(12)$ \\
\hline $\mathrm{C}(30)-\operatorname{Zr}(1)-\mathrm{H}(52)$ & $83.2(12)$ \\
\hline
\end{tabular}




\begin{tabular}{|c|c|}
\hline $\mathrm{C}(7)-\operatorname{Zr}(1)-\mathrm{H}(52)$ & $110.7(12)$ \\
\hline $\operatorname{Zr}(2)-\operatorname{Zr}(1)-\mathrm{H}(52)$ & $39.8(12)$ \\
\hline $\mathrm{H}(51)-\mathrm{Zr}(1)-\mathrm{H}(52)$ & $59.3(18)$ \\
\hline $\mathrm{N}(2)-\operatorname{Zr}(1)-\mathrm{H}(53)$ & $80.2(12)$ \\
\hline $\mathrm{N}(1)-\operatorname{Zr}(1)-\mathrm{H}(53)$ & $86.8(13)$ \\
\hline $\mathrm{C}(33)-\operatorname{Zr}(1)-\mathrm{H}(53)$ & $138.3(13)$ \\
\hline $\mathrm{C}(34)-\operatorname{Zr}(1)-\mathrm{H}(53)$ & $133.3(12)$ \\
\hline$C(32)-\operatorname{Zr}(1)-\mathrm{H}(53)$ & $158.1(12)$ \\
\hline $\mathrm{C}(31)-\operatorname{Zr}(1)-\mathrm{H}(53)$ & $168.1(13)$ \\
\hline$C(30)-\operatorname{Zr}(1)-\mathrm{H}(53)$ & $145.9(12)$ \\
\hline $\mathrm{C}(7)-\operatorname{Zr}(1)-\mathrm{H}(53)$ & $76.2(12)$ \\
\hline $\operatorname{Zr}(2)-\operatorname{Zr}(1)-\mathrm{H}(53)$ & $33.9(12)$ \\
\hline $\mathrm{H}(51)-\mathrm{Zr}(1)-\mathrm{H}(53)$ & $61.7(18)$ \\
\hline $\mathrm{H}(52)-\mathrm{Zr}(1)-\mathrm{H}(53)$ & $64.7(17)$ \\
\hline$N(4)-\operatorname{Zr}(2)-N(3)$ & $57.92(14)$ \\
\hline$N(4)-\operatorname{Zr}(2)-C(37)$ & $138.26(16)$ \\
\hline$N(3)-\operatorname{Zr}(2)-C(37)$ & $129.22(15)$ \\
\hline$N(4)-\operatorname{Zr}(2)-C(36)$ & $107.79(17)$ \\
\hline$N(3)-\operatorname{Zr}(2)-C(36)$ & $131.05(17)$ \\
\hline$C(37)-\operatorname{Zr}(2)-C(36)$ & $32.27(18)$ \\
\hline$N(4)-\operatorname{Zr}(2)-C(35)$ & $85.83(16)$ \\
\hline$N(3)-\operatorname{Zr}(2)-C(35)$ & $99.00(17)$ \\
\hline$C(37)-\operatorname{Zr}(2)-C(35)$ & $53.36(18)$ \\
\hline$C(36)-\operatorname{Zr}(2)-C(35)$ & $32.62(17)$ \\
\hline$N(4)-\operatorname{Zr}(2)-C(38)$ & $129.62(16)$ \\
\hline$N(3)-\operatorname{Zr}(2)-C(38)$ & $97.18(16)$ \\
\hline$C(37)-\operatorname{Zr}(2)-C(38)$ & $32.25(17)$ \\
\hline$C(36)-\operatorname{Zr}(2)-C(38)$ & $53.47(19)$ \\
\hline$C(35)-\operatorname{Zr}(2)-C(38)$ & $53.00(18)$ \\
\hline$N(4)-\operatorname{Zr}(2)-C(39)$ & $97.68(15)$ \\
\hline$N(3)-\operatorname{Zr}(2)-C(39)$ & $80.72(16)$ \\
\hline$C(37)-\operatorname{Zr}(2)-C(39)$ & $53.18(16)$ \\
\hline$C(36)-\operatorname{Zr}(2)-C(39)$ & $53.31(19)$ \\
\hline$C(35)-\operatorname{Zr}(2)-C(39)$ & $31.64(19)$ \\
\hline$C(38)-\operatorname{Zr}(2)-C(39)$ & $32.06(17)$ \\
\hline$N(4)-\operatorname{Zr}(2)-C(23)$ & $29.52(14)$ \\
\hline$N(3)-\operatorname{Zr}(2)-C(23)$ & $29.65(14)$ \\
\hline$C(37)-\operatorname{Zr}(2)-C(23)$ & $148.16(15)$ \\
\hline$C(36)-\operatorname{Zr}(2)-C(23)$ & $128.97(16)$ \\
\hline$C(35)-\operatorname{Zr}(2)-C(23)$ & $98.85(16)$ \\
\hline$C(38)-\operatorname{Zr}(2)-C(23)$ & $120.91(16)$ \\
\hline$C(39)-\operatorname{Zr}(2)-C(23)$ & $95.12(15)$ \\
\hline$N(4)-\operatorname{Zr}(2)-\operatorname{Zr}(1)$ & $117.27(10)$ \\
\hline$N(3)-\operatorname{Zr}(2)-\operatorname{Zr}(1)$ & $102.53(10)$ \\
\hline$C(37)-\operatorname{Zr}(2)-\operatorname{Zr}(1)$ & $101.77(12)$ \\
\hline$C(36)-\operatorname{Zr}(2)-\operatorname{Zr}(1)$ & $123.13(13)$ \\
\hline$C(35)-\operatorname{Zr}(2)-\operatorname{Zr}(1)$ & $154.49(13)$ \\
\hline$C(38)-\operatorname{Zr}(2)-\operatorname{Zr}(1)$ & $110.27(13)$ \\
\hline$C(39)-\operatorname{Zr}(2)-\operatorname{Zr}(1)$ & $140.93(13)$ \\
\hline$C(23)-\operatorname{Zr}(2)-\operatorname{Zr}(1)$ & $106.63(10)$ \\
\hline $\mathrm{N}(4)-\mathrm{Zr}(2)-\mathrm{H}(51)$ & $133.1(14)$ \\
\hline $\mathrm{N}(3)-\mathrm{Zr}(2)-\mathrm{H}(51)$ & $136.5(13)$ \\
\hline $\mathrm{C}(37)-\mathrm{Zr}(2)-\mathrm{H}(51)$ & $74.3(14)$ \\
\hline $\mathrm{C}(36)-\mathrm{Zr}(2)-\mathrm{H}(51)$ & $89.2(14)$ \\
\hline$C(35)-Z r(2)-H(51)$ & $121.7(14)$ \\
\hline $\mathrm{C}(38)-\mathrm{Zr}(2)-\mathrm{H}(51)$ & $95.9(14)$ \\
\hline $\mathrm{C}(39)-\mathrm{Zr}(2)-\mathrm{H}(51)$ & $126.1(14)$ \\
\hline
\end{tabular}




\begin{tabular}{|c|c|}
\hline $\mathrm{C}(23)-\operatorname{Zr}(2)-\mathrm{H}(51)$ & $137.3(14)$ \\
\hline $\operatorname{Zr}(1)-\operatorname{Zr}(2)-\mathrm{H}(51)$ & $34.4(14)$ \\
\hline $\mathrm{N}(4)-\operatorname{Zr}(2)-\mathrm{H}(52)$ & $132.3(11)$ \\
\hline $\mathrm{N}(3)-\operatorname{Zr}(2)-\mathrm{H}(52)$ & $86.4(11)$ \\
\hline $\mathrm{C}(37)-\mathrm{Zr}(2)-\mathrm{H}(52)$ & $88.2(11)$ \\
\hline$C(36)-\operatorname{Zr}(2)-H(52)$ & $119.8(11)$ \\
\hline$C(35)-\operatorname{Zr}(2)-H(52)$ & $134.1(11)$ \\
\hline$C(38)-\operatorname{Zr}(2)-\mathrm{H}(52)$ & $81.1(11)$ \\
\hline $\mathrm{C}(39)-\operatorname{Zr}(2)-\mathrm{H}(52)$ & $107.2(11$ \\
\hline$C(23)-\operatorname{Zr}(2)-\mathrm{H}(52)$ & $106.7(11$ \\
\hline $\operatorname{Zr}(1)-\operatorname{Zr}(2)-\mathrm{H}(52)$ & $35.8(11)$ \\
\hline $\mathrm{H}(51)-\mathrm{Zr}(2)-\mathrm{H}(52)$ & $55.2(16$ \\
\hline$N(4)-\operatorname{Zr}(2)-H(53)$ & $83.2(12)$ \\
\hline $\mathrm{N}(3)-\operatorname{Zr}(2)-\mathrm{H}(53)$ & $85.0(12)$ \\
\hline$C(37)-\operatorname{Zr}(2)-\mathrm{H}(53)$ & $134.2(12)$ \\
\hline$C(36)-\operatorname{Zr}(2)-\mathrm{H}(53)$ & $143.1(12)$ \\
\hline$C(35)-\operatorname{Zr}(2)-\mathrm{H}(53)$ & $164.0(13)$ \\
\hline$C(38)-\operatorname{Zr}(2)-\mathrm{H}(53)$ & $142.2(13)$ \\
\hline$C(39)-\operatorname{Zr}(2)-\mathrm{H}(53)$ & $162.4(13)$ \\
\hline$C(23)-\operatorname{Zr}(2)-\mathrm{H}(53)$ & $76.9(12)$ \\
\hline $\operatorname{Zr}(1)-\operatorname{Zr}(2)-\mathrm{H}(53)$ & $34.1(12)$ \\
\hline $\mathrm{H}(51)-\mathrm{Zr}(2)-\mathrm{H}(53)$ & $60.4(18)$ \\
\hline $\mathrm{H}(52)-\mathrm{Zr}(2)-\mathrm{H}(53)$ & $61.4(16)$ \\
\hline $\mathrm{N}(4)-\operatorname{Zr}(2)-\mathrm{H}(54)$ & $73.8(18)$ \\
\hline $\mathrm{N}(3)-\operatorname{Zr}(2)-\mathrm{H}(54)$ & $130.0(18)$ \\
\hline $\mathrm{C}(37)-\mathrm{Zr}(2)-\mathrm{H}(54)$ & $94.9(18)$ \\
\hline$C(36)-\operatorname{Zr}(2)-\mathrm{H}(54)$ & $73.2(18)$ \\
\hline $\mathrm{C}(35)-\mathrm{Zr}(2)-\mathrm{H}(54)$ & $89.1(18)$ \\
\hline $\mathrm{C}(38)-\mathrm{Zr}(2)-\mathrm{H}(54)$ & $125.3(18)$ \\
\hline $\mathrm{C}(39)-\mathrm{Zr}(2)-\mathrm{H}(54)$ & $120.5(18)$ \\
\hline$C(23)-\operatorname{Zr}(2)-\mathrm{H}(54)$ & $100.4(18)$ \\
\hline $\operatorname{Zr}(1)-\operatorname{Zr}(2)-\mathrm{H}(54)$ & $87.5(18)$ \\
\hline $\mathrm{H}(51)-\mathrm{Zr}(2)-\mathrm{H}(54)$ & $70(2)$ \\
\hline $\mathrm{H}(52)-\mathrm{Zr}(2)-\mathrm{H}(54)$ & $121.9(19)$ \\
\hline $\mathrm{H}(53)-\mathrm{Zr}(2)-\mathrm{H}(54)$ & $77(2)$ \\
\hline$C(13)-O(1)-C(17)$ & $118.2(4)$ \\
\hline$C(7)-N(1)-C(2)$ & $123.9(4)$ \\
\hline $\mathrm{C}(7)-\mathrm{N}(1)-\operatorname{Zr}(1)$ & $90.8(3)$ \\
\hline $\mathrm{C}(2)-\mathrm{N}(1)-\operatorname{Zr}(1)$ & $142.6(3)$ \\
\hline$C(7)-N(2)-C(5)$ & $124.2(4)$ \\
\hline$C(7)-N(2)-\operatorname{Zr}(1)$ & $92.5(3)$ \\
\hline$C(5)-N(2)-\operatorname{Zr}(1)$ & $132.7(3)$ \\
\hline$C(23)-N(3)-C(25)$ & $124.8(4)$ \\
\hline$C(23)-N(3)-\operatorname{Zr}(2)$ & $92.1(3)$ \\
\hline$C(25)-N(3)-\operatorname{Zr}(2)$ & $137.8(3)$ \\
\hline $\mathrm{C}(23)-\mathrm{N}(4)-\mathrm{C}(28)$ & $121.8(4)$ \\
\hline$C(23)-N(4)-\operatorname{Zr}(2)$ & $93.1(3)$ \\
\hline$C(28)-N(4)-\operatorname{Zr}(2)$ & $139.2(3)$ \\
\hline $\mathrm{C}(2)-\mathrm{C}(1)-\mathrm{H}(1 \mathrm{~A})$ & 109.5 \\
\hline $\mathrm{C}(2)-\mathrm{C}(1)-\mathrm{H}(1 \mathrm{~B})$ & 109.5 \\
\hline $\mathrm{H}(1 \mathrm{~A})-\mathrm{C}(1)-\mathrm{H}(1 \mathrm{~B})$ & 109.5 \\
\hline $\mathrm{C}(2)-\mathrm{C}(1)-\mathrm{H}(1 \mathrm{C})$ & 109.5 \\
\hline $\mathrm{H}(1 \mathrm{~A})-\mathrm{C}(1)-\mathrm{H}(1 \mathrm{C})$ & 109.5 \\
\hline $\mathrm{H}(1 \mathrm{~B})-\mathrm{C}(1)-\mathrm{H}(1 \mathrm{C})$ & 109.5 \\
\hline $\mathrm{N}(1)-\mathrm{C}(2)-\mathrm{C}(3)$ & $108.5(4)$ \\
\hline $\mathrm{N}(1)-\mathrm{C}(2)-\mathrm{C}(1)$ & $111.9(4)$ \\
\hline$C(3)-C(2)-C(1)$ & $109.6(4)$ \\
\hline
\end{tabular}




\begin{tabular}{|c|c|}
\hline $\mathrm{N}(1)-\mathrm{C}(2)-\mathrm{H}(2)$ & 108.9 \\
\hline $\mathrm{C}(3)-\mathrm{C}(2)-\mathrm{H}(2)$ & 108.9 \\
\hline $\mathrm{C}(1)-\mathrm{C}(2)-\mathrm{H}(2)$ & 108.9 \\
\hline$C(2)-C(3)-H(3 A)$ & 109.5 \\
\hline$C(2)-C(3)-H(3 B)$ & 109.5 \\
\hline $\mathrm{H}(3 \mathrm{~A})-\mathrm{C}(3)-\mathrm{H}(3 \mathrm{~B})$ & 109.5 \\
\hline $\mathrm{C}(2)-\mathrm{C}(3)-\mathrm{H}(3 \mathrm{C})$ & 109.5 \\
\hline $\mathrm{H}(3 \mathrm{~A})-\mathrm{C}(3)-\mathrm{H}(3 \mathrm{C})$ & 109.5 \\
\hline $\mathrm{H}(3 \mathrm{~B})-\mathrm{C}(3)-\mathrm{H}(3 \mathrm{C})$ & 109.5 \\
\hline $\mathrm{C}(5)-\mathrm{C}(4)-\mathrm{H}(4 \mathrm{~A})$ & 109.5 \\
\hline$C(5)-C(4)-H(4 B)$ & 109.5 \\
\hline $\mathrm{H}(4 \mathrm{~A})-\mathrm{C}(4)-\mathrm{H}(4 \mathrm{~B})$ & 109.5 \\
\hline $\mathrm{C}(5)-\mathrm{C}(4)-\mathrm{H}(4 \mathrm{C})$ & 109.5 \\
\hline $\mathrm{H}(4 \mathrm{~A})-\mathrm{C}(4)-\mathrm{H}(4 \mathrm{C})$ & 109.5 \\
\hline $\mathrm{H}(4 \mathrm{~B})-\mathrm{C}(4)-\mathrm{H}(4 \mathrm{C})$ & 109.5 \\
\hline$N(2)-C(5)-C(4)$ & $111.9(4)$ \\
\hline$N(2)-C(5)-C(6)$ & $107.4(4)$ \\
\hline$C(4)-C(5)-C(6)$ & $109.7(4)$ \\
\hline $\mathrm{N}(2)-\mathrm{C}(5)-\mathrm{H}(5)$ & 109.3 \\
\hline $\mathrm{C}(4)-\mathrm{C}(5)-\mathrm{H}(5)$ & 109.3 \\
\hline $\mathrm{C}(6)-\mathrm{C}(5)-\mathrm{H}(5)$ & 109.3 \\
\hline$C(5)-C(6)-H(6 A)$ & 109.5 \\
\hline$C(5)-C(6)-H(6 B)$ & 109.5 \\
\hline $\mathrm{H}(6 \mathrm{~A})-\mathrm{C}(6)-\mathrm{H}(6 \mathrm{~B})$ & 109.5 \\
\hline$C(5)-C(6)-H(6 C)$ & 109.5 \\
\hline $\mathrm{H}(6 \mathrm{~A})-\mathrm{C}(6)-\mathrm{H}(6 \mathrm{C})$ & 109.5 \\
\hline $\mathrm{H}(6 \mathrm{~B})-\mathrm{C}(6)-\mathrm{H}(6 \mathrm{C})$ & 109.5 \\
\hline $\mathrm{N}(1)-\mathrm{C}(7)-\mathrm{N}(2)$ & $112.6(4)$ \\
\hline $\mathrm{N}(1)-\mathrm{C}(7)-\mathrm{C}(8)$ & $124.2(4)$ \\
\hline$N(2)-C(7)-C(8)$ & $123.2(5)$ \\
\hline$N(1)-C(7)-\operatorname{Zr}(1)$ & $58.8(2)$ \\
\hline$N(2)-C(7)-\operatorname{Zr}(1)$ & $57.0(2)$ \\
\hline$C(8)-C(7)-\operatorname{Zr}(1)$ & $163.3(3)$ \\
\hline$C(9)-C(8)-C(13)$ & $118.8(4)$ \\
\hline$C(9)-C(8)-C(7)$ & $118.1(4)$ \\
\hline$C(13)-C(8)-C(7)$ & $123.1(4)$ \\
\hline$C(8)-C(9)-C(10)$ & $119.9(4)$ \\
\hline $\mathrm{C}(8)-\mathrm{C}(9)-\mathrm{H}(9)$ & 120.0 \\
\hline $\mathrm{C}(10)-\mathrm{C}(9)-\mathrm{H}(9)$ & 120.0 \\
\hline$C(11)-C(10)-C(9)$ & $120.3(4)$ \\
\hline $\mathrm{C}(11)-\mathrm{C}(10)-\mathrm{H}(10)$ & 119.8 \\
\hline $\mathrm{C}(9)-\mathrm{C}(10)-\mathrm{H}(10)$ & 119.8 \\
\hline$C(10)-C(11)-C(12)$ & $121.5(5)$ \\
\hline $\mathrm{C}(10)-\mathrm{C}(11)-\mathrm{H}(11)$ & 119.2 \\
\hline $\mathrm{C}(12)-\mathrm{C}(11)-\mathrm{H}(11)$ & 119.2 \\
\hline$C(13)-C(12)-C(11)$ & $118.3(4)$ \\
\hline$C(13)-C(12)-C(15)$ & $121.5(4)$ \\
\hline$C(11)-C(12)-C(15)$ & $120.1(4)$ \\
\hline $\mathrm{O}(1)-\mathrm{C}(13)-\mathrm{C}(12)$ & $123.6(4)$ \\
\hline $\mathrm{O}(1)-\mathrm{C}(13)-\mathrm{C}(8)$ & $115.3(4)$ \\
\hline$C(12)-C(13)-C(8)$ & $121.1(4)$ \\
\hline $\mathrm{C}(15)-\mathrm{C}(14)-\mathrm{H}(14 \mathrm{~A})$ & 109.5 \\
\hline $\mathrm{C}(15)-\mathrm{C}(14)-\mathrm{H}(14 \mathrm{~B})$ & 109.5 \\
\hline $\mathrm{H}(14 \mathrm{~A})-\mathrm{C}(14)-\mathrm{H}(14 \mathrm{~B})$ & 109.5 \\
\hline $\mathrm{C}(15)-\mathrm{C}(14)-\mathrm{H}(14 \mathrm{C})$ & 109.5 \\
\hline $\mathrm{H}(14 \mathrm{~A})-\mathrm{C}(14)-\mathrm{H}(14 \mathrm{C})$ & 109.5 \\
\hline $\mathrm{H}(14 \mathrm{~B})-\mathrm{C}(14)-\mathrm{H}(14 \mathrm{C})$ & 109.5 \\
\hline
\end{tabular}




\begin{tabular}{|c|c|}
\hline$C(18)-C(15)-C(14)$ & $109.7(4)$ \\
\hline$C(18)-C(15)-C(12)$ & $110.3(4)$ \\
\hline$C(14)-C(15)-C(12)$ & $109.4(4)$ \\
\hline$C(18)-C(15)-C(16)$ & $109.7(4)$ \\
\hline$C(14)-C(15)-C(16)$ & $109.4(4)$ \\
\hline$C(12)-C(15)-C(16)$ & $108.3(4)$ \\
\hline $\mathrm{C}(15)-\mathrm{C}(16)-\mathrm{H}(16 \mathrm{~A})$ & 109.5 \\
\hline $\mathrm{C}(15)-\mathrm{C}(16)-\mathrm{H}(16 \mathrm{~B})$ & 109.5 \\
\hline $\mathrm{H}(16 \mathrm{~A})-\mathrm{C}(16)-\mathrm{H}(16 \mathrm{~B})$ & 109.5 \\
\hline $\mathrm{C}(15)-\mathrm{C}(16)-\mathrm{H}(16 \mathrm{C})$ & 109.5 \\
\hline $\mathrm{H}(16 \mathrm{~A})-\mathrm{C}(16)-\mathrm{H}(16 \mathrm{C})$ & 109.5 \\
\hline $\mathrm{H}(16 \mathrm{~B})-\mathrm{C}(16)-\mathrm{H}(16 \mathrm{C})$ & 109.5 \\
\hline $\mathrm{C}(18)-\mathrm{C}(17)-\mathrm{O}(1)$ & $123.1(4)$ \\
\hline$C(18)-C(17)-C(22)$ & $122.1(4)$ \\
\hline $\mathrm{O}(1)-\mathrm{C}(17)-\mathrm{C}(22)$ & $114.8(4)$ \\
\hline$C(17)-C(18)-C(19)$ & $117.4(4)$ \\
\hline$C(17)-C(18)-C(15)$ & $122.8(4)$ \\
\hline$C(19)-C(18)-C(15)$ & $119.8(4)$ \\
\hline$C(20)-C(19)-C(18)$ & $121.9(5)$ \\
\hline $\mathrm{C}(20)-\mathrm{C}(19)-\mathrm{H}(19)$ & 119.0 \\
\hline $\mathrm{C}(18)-\mathrm{C}(19)-\mathrm{H}(19)$ & 119.0 \\
\hline$C(21)-C(20)-C(19)$ & $119.5(5)$ \\
\hline$C(21)-C(20)-H(20)$ & 120.3 \\
\hline $\mathrm{C}(19)-\mathrm{C}(20)-\mathrm{H}(20)$ & 120.3 \\
\hline$C(20)-C(21)-C(22)$ & $120.8(5)$ \\
\hline $\mathrm{C}(20)-\mathrm{C}(21)-\mathrm{H}(21)$ & 119.6 \\
\hline $\mathrm{C}(22)-\mathrm{C}(21)-\mathrm{H}(21)$ & 119.6 \\
\hline$C(17)-C(22)-C(21)$ & $118.3(4)$ \\
\hline$C(17)-C(22)-C(23)$ & $123.6(4)$ \\
\hline$C(21)-C(22)-C(23)$ & $118.1(4)$ \\
\hline$N(4)-C(23)-N(3)$ & $112.2(4)$ \\
\hline$N(4)-C(23)-C(22)$ & $122.6(4)$ \\
\hline$N(3)-C(23)-C(22)$ & $125.2(4)$ \\
\hline$N(4)-C(23)-\operatorname{Zr}(2)$ & $57.4(2)$ \\
\hline$N(3)-C(23)-\operatorname{Zr}(2)$ & $58.2(2)$ \\
\hline$C(22)-C(23)-\operatorname{Zr}(2)$ & $162.7(3)$ \\
\hline$C(25)-C(24)-H(24 A)$ & 109.5 \\
\hline$C(25)-C(24)-H(24 B)$ & 109.5 \\
\hline $\mathrm{H}(24 \mathrm{~A})-\mathrm{C}(24)-\mathrm{H}(24 \mathrm{~B})$ & 109.5 \\
\hline $\mathrm{C}(25)-\mathrm{C}(24)-\mathrm{H}(24 \mathrm{C})$ & 109.5 \\
\hline $\mathrm{H}(24 \mathrm{~A})-\mathrm{C}(24)-\mathrm{H}(24 \mathrm{C})$ & 109.5 \\
\hline $\mathrm{H}(24 \mathrm{~B})-\mathrm{C}(24)-\mathrm{H}(24 \mathrm{C})$ & 109.5 \\
\hline$N(3)-C(25)-C(26)$ & $109.0(4)$ \\
\hline$N(3)-C(25)-C(24)$ & $114.4(4)$ \\
\hline$C(26)-C(25)-C(24)$ & $107.9(4)$ \\
\hline $\mathrm{N}(3)-\mathrm{C}(25)-\mathrm{H}(25)$ & 108.4 \\
\hline$C(26)-C(25)-H(25)$ & 108.4 \\
\hline $\mathrm{C}(24)-\mathrm{C}(25)-\mathrm{H}(25)$ & 108.4 \\
\hline$C(25)-C(26)-H(26 A)$ & 109.5 \\
\hline$C(25)-C(26)-H(26 B)$ & 109.5 \\
\hline $\mathrm{H}(26 \mathrm{~A})-\mathrm{C}(26)-\mathrm{H}(26 \mathrm{~B})$ & 109.5 \\
\hline$C(25)-C(26)-H(26 C)$ & 109.5 \\
\hline $\mathrm{H}(26 \mathrm{~A})-\mathrm{C}(26)-\mathrm{H}(26 \mathrm{C})$ & 109.5 \\
\hline $\mathrm{H}(26 \mathrm{~B})-\mathrm{C}(26)-\mathrm{H}(26 \mathrm{C})$ & 109.5 \\
\hline $\mathrm{C}(28)-\mathrm{C}(27)-\mathrm{H}(27 \mathrm{~A})$ & 109.5 \\
\hline $\mathrm{C}(28)-\mathrm{C}(27)-\mathrm{H}(27 \mathrm{~B})$ & 109.5 \\
\hline $\mathrm{H}(27 \mathrm{~A})-\mathrm{C}(27)-\mathrm{H}(27 \mathrm{~B})$ & 109.5 \\
\hline
\end{tabular}




\begin{tabular}{|c|c|}
\hline $\mathrm{C}(28)-\mathrm{C}(27)-\mathrm{H}(27 \mathrm{C})$ & 109.5 \\
\hline $\mathrm{H}(27 \mathrm{~A})-\mathrm{C}(27)-\mathrm{H}(27 \mathrm{C})$ & 109.5 \\
\hline $\mathrm{H}(27 \mathrm{~B})-\mathrm{C}(27)-\mathrm{H}(27 \mathrm{C})$ & 109.5 \\
\hline$N(4)-C(28)-C(27)$ & $111.6(4)$ \\
\hline $\mathrm{N}(4)-\mathrm{C}(28)-\mathrm{C}(29)$ & $107.8(4)$ \\
\hline$C(27)-C(28)-C(29)$ & $111.4(4)$ \\
\hline $\mathrm{N}(4)-\mathrm{C}(28)-\mathrm{H}(28)$ & 108.7 \\
\hline $\mathrm{C}(27)-\mathrm{C}(28)-\mathrm{H}(28)$ & 108.7 \\
\hline $\mathrm{C}(29)-\mathrm{C}(28)-\mathrm{H}(28)$ & 108.7 \\
\hline $\mathrm{C}(28)-\mathrm{C}(29)-\mathrm{H}(29 \mathrm{~A})$ & 109.5 \\
\hline $\mathrm{C}(28)-\mathrm{C}(29)-\mathrm{H}(29 \mathrm{~B})$ & 109.5 \\
\hline $\mathrm{H}(29 \mathrm{~A})-\mathrm{C}(29)-\mathrm{H}(29 \mathrm{~B})$ & 109.5 \\
\hline $\mathrm{C}(28)-\mathrm{C}(29)-\mathrm{H}(29 \mathrm{C})$ & 109.5 \\
\hline $\mathrm{H}(29 \mathrm{~A})-\mathrm{C}(29)-\mathrm{H}(29 \mathrm{C})$ & 109.5 \\
\hline $\mathrm{H}(29 B)-\mathrm{C}(29)-\mathrm{H}(29 \mathrm{C})$ & 109.5 \\
\hline$C(34)-C(30)-C(31)$ & $108.1(5)$ \\
\hline$C(34)-C(30)-\operatorname{Zr}(1)$ & $73.7(3)$ \\
\hline$C(31)-C(30)-\operatorname{Zr}(1)$ & $74.0(3)$ \\
\hline $\mathrm{C}(34)-\mathrm{C}(30)-\mathrm{H}(30)$ & 126.0 \\
\hline $\mathrm{C}(31)-\mathrm{C}(30)-\mathrm{H}(30)$ & 126.0 \\
\hline $\operatorname{Zr}(1)-\mathrm{C}(30)-\mathrm{H}(30)$ & 118.3 \\
\hline$C(30)-C(31)-C(32)$ & $108.9(5)$ \\
\hline$C(30)-C(31)-\operatorname{Zr}(1)$ & $74.0(3)$ \\
\hline$C(32)-C(31)-\operatorname{Zr}(1)$ & $73.8(3)$ \\
\hline $\mathrm{C}(30)-\mathrm{C}(31)-\mathrm{H}(31)$ & 125.5 \\
\hline $\mathrm{C}(32)-\mathrm{C}(31)-\mathrm{H}(31)$ & 125.5 \\
\hline $\operatorname{Zr}(1)-\mathrm{C}(31)-\mathrm{H}(31)$ & 118.5 \\
\hline$C(31)-C(32)-C(33)$ & $107.3(5)$ \\
\hline$C(31)-C(32)-\operatorname{Zr}(1)$ & $74.1(3)$ \\
\hline$C(33)-C(32)-\operatorname{Zr}(1)$ & $73.4(3)$ \\
\hline $\mathrm{C}(31)-\mathrm{C}(32)-\mathrm{H}(32)$ & 126.3 \\
\hline $\mathrm{C}(33)-\mathrm{C}(32)-\mathrm{H}(32)$ & 126.3 \\
\hline $\operatorname{Zr}(1)-\mathrm{C}(32)-\mathrm{H}(32)$ & 118.3 \\
\hline$C(32)-C(33)-C(34)$ & $107.8(6)$ \\
\hline$C(32)-C(33)-\operatorname{Zr}(1)$ & $74.2(3)$ \\
\hline$C(34)-C(33)-\operatorname{Zr}(1)$ & $73.9(3)$ \\
\hline $\mathrm{C}(32)-\mathrm{C}(33)-\mathrm{H}(33)$ & 126.1 \\
\hline $\mathrm{C}(34)-\mathrm{C}(33)-\mathrm{H}(33)$ & 126.1 \\
\hline $\operatorname{Zr}(1)-\mathrm{C}(33)-\mathrm{H}(33)$ & 117.7 \\
\hline$C(30)-C(34)-C(33)$ & $107.9(5)$ \\
\hline$C(30)-C(34)-\operatorname{Zr}(1)$ & $74.3(3)$ \\
\hline$C(33)-C(34)-\operatorname{Zr}(1)$ & $73.4(3)$ \\
\hline $\mathrm{C}(30)-\mathrm{C}(34)-\mathrm{H}(34)$ & 126.1 \\
\hline $\mathrm{C}(33)-\mathrm{C}(34)-\mathrm{H}(34)$ & 126.1 \\
\hline $\operatorname{Zr}(1)-\mathrm{C}(34)-\mathrm{H}(34)$ & 118.2 \\
\hline$C(39)-C(35)-C(36)$ & $108.6(5)$ \\
\hline$C(39)-C(35)-\operatorname{Zr}(2)$ & $75.2(3)$ \\
\hline$C(36)-C(35)-\operatorname{Zr}(2)$ & $73.4(3)$ \\
\hline $\mathrm{C}(39)-\mathrm{C}(35)-\mathrm{H}(35)$ & 125.7 \\
\hline $\mathrm{C}(36)-\mathrm{C}(35)-\mathrm{H}(35)$ & 125.7 \\
\hline $\operatorname{Zr}(2)-\mathrm{C}(35)-\mathrm{H}(35)$ & 117.6 \\
\hline$C(37)-C(36)-C(35)$ & $107.0(6)$ \\
\hline$C(37)-C(36)-\operatorname{Zr}(2)$ & $73.5(3)$ \\
\hline$C(35)-C(36)-\operatorname{Zr}(2)$ & $74.0(3)$ \\
\hline $\mathrm{C}(37)-\mathrm{C}(36)-\mathrm{H}(36)$ & 126.5 \\
\hline $\mathrm{C}(35)-\mathrm{C}(36)-\mathrm{H}(36)$ & 126.5 \\
\hline $\operatorname{Zr}(2)-\mathrm{C}(36)-\mathrm{H}(36)$ & 118.0 \\
\hline
\end{tabular}




$\begin{array}{lc}C(36)-C(37)-C(38) & 108.6(5) \\ C(36)-C(37)-\operatorname{Zr}(2) & 74.2(3) \\ C(38)-C(37)-\operatorname{Zr}(2) & 74.9(3) \\ C(36)-C(37)-H(37) & 125.7 \\ C(38)-C(37)-H(37) & 125.7 \\ \operatorname{Zr}(2)-C(37)-H(37) & 117.2 \\ C(37)-C(38)-C(39) & 107.7(5) \\ C(37)-C(38)-\operatorname{Zr}(2) & 72.9(3) \\ C(39)-C(38)-\operatorname{Zr}(2) & 74.7(3) \\ C(37)-C(38)-H(38) & 126.1 \\ C(39)-C(38)-H(38) & 126.1 \\ \text { Zr(2)-C (38)-H(38) } & 118.3 \\ C(35)-C(39)-C(38) & 108.1(5) \\ C(35)-C(39)-\operatorname{Zr}(2) & 73.1(3) \\ C(38)-C(39)-\operatorname{Zr}(2) & 73.3(3) \\ C(35)-C(39)-H(39) & 126.0 \\ C(38)-C(39)-H(39) & 126.0 \\ \text { Zr }(2)-C(39)-H(39) & 119.5\end{array}$

